\title{
Self-consistent theory of current and voltage noise in multimode ballistic conductors
}

\author{
O. M. Bulashenko* and J. M. Rubí \\ Departament de Física Fonamental, Universitat de Barcelona, Diagonal 647, E-08028 Barcelona, Spain
}

(Received 12 January 2002; published 18 July 2002)

\begin{abstract}
Electron transport in a self-consistent potential along a ballistic two-terminal conductor has been investigated. We have derived general formulas which describe the nonlinear current-voltage characteristics, differential conductance, and low-frequency current and voltage noise assuming an arbitrary distribution function and correlation properties of injected electrons. The analytical results have been obtained for a wide range of biases: from equilibrium to high values beyond the linear-response regime. The particular case of a threedimensional Fermi-Dirac injection has been analyzed. We show that the Coulomb correlations are manifested in the negative excess voltage noise, i.e., the voltage fluctuations under high-field transport conditions can be less than in equilibrium.
\end{abstract}

DOI: 10.1103/PhysRevB.66.045310

PACS number(s): 73.50.Td

\section{INTRODUCTION}

Recently, measurements of nonequilibrium noise have emerged as a fundamental tool to obtain information on the transport properties and interactions among carriers in mesoscopic systems. ${ }^{1-3}$ Shot-noise suppression in ballistic conductors caused by Fermi correlations has been studied extensively both theoretically ${ }^{4-6}$ and experimentally. ${ }^{7-10}$ Within the scattering approach, it is usually assumed that the ballistic (phase-coherent) conductor is attached to reservoirs (terminals or leads) with different chemical potentials. In this approach, the mean current in a two-terminal conductor is given by

$$
I=\frac{q}{2 \pi \hbar} \sum_{n} \int d \varepsilon \mathcal{T}_{n}(\varepsilon)\left[f_{L}(\varepsilon)-f_{R}(\varepsilon)\right],
$$

where $q$ is the electron charge, $f_{L, R}(\varepsilon)$ the energy distribution functions at the left $(\mathrm{L})$ and right $(\mathrm{R})$ reservoirs, and $\mathcal{T}_{n}$ the transmission probabilities associated with $n$ transverse quantum modes (channels). ${ }^{3}$ The corresponding currentnoise power at zero-frequency has been obtained in the form $^{4,5}$ (also see Refs. 3, 6, and 11)

$$
\begin{aligned}
S_{I}= & \frac{q^{2}}{\pi \hbar} \sum_{n} \int d \varepsilon\left\{\mathcal{T}_{n}(\varepsilon)\left[f_{L}\left(1-f_{L}\right)+f_{R}\left(1-f_{R}\right)\right]\right. \\
& \left.+\mathcal{T}_{n}(\varepsilon)\left[1-\mathcal{T}_{n}(\varepsilon)\right]\left(f_{L}-f_{R}\right)^{2}\right\} .
\end{aligned}
$$

In Eq. (2) the noise is a combination of the thermal emission noise of the reservoirs and of the partition noise appeared due to the current partitioning between the incoming and outgoing states [scattering on tunneling barrier(s), elastic scatterer(s), point contact(s), etc.]. Although in some limits the well-known noise terms can be identified (associated with thermal noise or shot noise), they cannot be separated in general. Out of equilibrium, the noise can manifest itself in a different way, depending on the conditions. At low biases $U$, Eq. (2) gives the partition shot noise-the excess noise linear in current (bias), which does not vanish at zero temperature. It is suppressed below the Poisson $2 q I$ value approximately by the factor ${ }^{3} \Sigma_{n} \mathcal{T}_{n}\left(1-\mathcal{T}_{n}\right) / \Sigma_{n} \mathcal{T}_{n}$. This type of excess noise appears whenever there is a partitioning of cur- rent $\left(\mathcal{T}_{n} \neq 0 ; 1\right)$. It vanishes for fully ballistic systems for which there is no partitioning $\left(\mathcal{T}_{n}=1\right.$ ) (see experimental evidence ${ }^{7-10}$ ). In the absence of partitioning, the excess noise is in general no longer linear in the current. The inherent randomness in the emission of carriers from the reservoirs is at the origin of this type of nonequilibrium noise. Presumably, it is more pronounced for sufficiently high biases when $f_{R} \ll f_{L}$ and the transmission dominates in only one direction. In this case, noise formula (2) is simplified to ${ }^{5}$

$$
S_{I}=\frac{q^{2}}{\pi \hbar} \sum_{n *} \int f_{L}\left(1-f_{L}\right) d \varepsilon
$$

where the summation is taken for open channels. ${ }^{12}$ For low electron densities the occupation numbers are small, $f_{L} \ll 1$, and Eq. (3) leads to the Schottky formula $S_{I}$ $=\left(q^{2} / \pi \hbar\right) \Sigma_{n} * \int f_{L} d \varepsilon=2 q I_{e m}$, where $I_{e m}$ is the emission current from the reservoir (vacuum-tube-like shot noise). In this low-density limit, shot noise is Poissonian since the transmission of carriers is uncorrelated. The factor $\left(1-f_{L}\right)$ in Eq. (3) introduces the Fermi correlations among carriers when the occupation numbers are not small in respect to 1 . This leads to the suppressed value of the shot noise. Note the difference between the partition shot noise mentioned earlier, and the emission shot noise given by Eq. (3). The former persists at zero temperature, since it reflects the granularity in charge transmission manifested by partitioning, while the latter vanishes at $T \rightarrow 0$, because its origin is the thermal fluctuations of the occupation numbers in the reservoirs. Indeed, at equilibrium the sum of two opposite shot-noise terms [Eq. (3)] gives the Nyquist formula. ${ }^{13}$

It should be stressed that both Eqs. (2) and (3) are not complete, since they ignore Coulomb interactions. The electrons are charged entities and, while moving along the conductor, they affect the electric potential giving rise to inhomogeneity. ${ }^{14}$ The self-consistent coupling between the nonhomogeneous electron density and potential landscape is very important to adequately describe the transport and noise under nonlinear far-from-equilibrium conditions. ${ }^{15,16}$ An interesting question is how the self-consistency may affect the current and noise formulas (1)-(3)? First, the transmission probabilities $\mathcal{T}_{n}$ for both current and noise become function- 


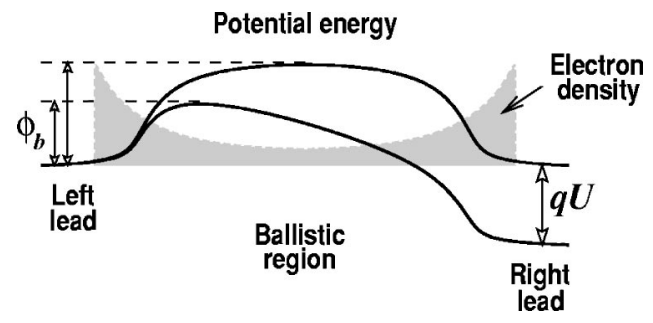

FIG. 1. Energy diagram determining the potential-barrier shape for a ballistic two-terminal conductor at equilibrium and under applied bias $U$. Barrier height $\Phi_{b}$, as seen from the left lead, decreases with bias. The filled area illustrates the nonhomogeneous electron-density distribution.

als of the time-averaged self-consistent potential profile $\bar{\varphi} \cdot{ }^{17}$ Second, in the current-noise formulas (2) and (3), which reflect only the injected current fluctuations $\delta I_{\text {inj }}$, the additional terms should appear caused by the current fluctuations $\delta I_{\text {ind }}$ induced by the fluctuations of the potential $\delta \varphi$. Finding the fluctuations $\delta \varphi$ is a complicated problem in general, since they are self-consistently linked to the fluctuations of the occupation numbers along the conductor. (The latter may be expressed through the fluctuations of the occupation numbers at the terminals, since the system is ballistic. ${ }^{18}$ ) As a result of this self-consistent coupling, the long-range Coulomb correlations appear, which may result in the noise suppression. ${ }^{6,19,20}$ It is believed, however, that such Coulomb correlations need to be taken into account for the description of systems in time-dependent external fields, or finitefrequency fluctuation spectra in stationary fields, while the zero-frequency fluctuations in stationary fields are not affected by them. ${ }^{3}$ We show that this is not always true. In the example we address in this paper, the additional terms induced by the self-consistent field are of the order of the fluctuations injected from the leads and cannot be neglected even in the zero-frequency limit at time-independent biases. Moreover, they can almost completely compensate the injected fluctuations up to an arbitrarily small value. At the same time, the gauge invariance required for the charge conservation is fulfilled. We also found that this Coulomb suppression of noise is manifested in the negative excess voltage noise. Current or voltage fluctuations in equilibrium, described by the fluctuation-dissipation theorem, usually increase when an external electric field is applied. We have an interesting example when an interacting (via Coulomb forces) electron system is less noisy at far-from-equilibrium conditions than in equilibrium. For noninteracting systems, such examples have been given by Lesovik and Loosen. ${ }^{21}$

To support our statement we present a theory of current and voltage fluctuations in a ballistic two-terminal conductor in a self-consistent field (Fig. 1). The calculation of the selfconsistent fluctuating field is, in general, a multidimensional problem which includes the electrostatic environment. For simplicity, we consider rather thick samples that allow us to use one-dimensional plane geometry for electrostatics. On the other hand, for wide conductors the number of transversal modes is large and the semiclassical treatment is sufficient. By assuming that there is no current partitioning $\left(\mathcal{T}_{n}\right.$ $=1$ for all the transmitting modes), we focus mainly on the nonequilibrium noise caused by thermal emission from the reservoirs under the action of the long-range Coulomb correlations inside the ballistic region, rather than on the partition shot noise. It should be noted that the previous theoretical studies have been devoted to ballistic conductors with a small number of quantum modes (quantum point contacts) with the Fermi suppression of the partition shot noise (Coulomb correlations have been ignored). ${ }^{4-6,22}$

The main results of the present investigation are as follows: We have obtained complete analytical expressions for the steady-state spatial profiles (carrier density, selfconsistent field), mean current, and differential conductance, as well as the current and voltage noise powers in ballistic multimode conductors. The analytical results have been obtained for a wide range of biases: from equilibrium to high values beyond the linear-response regime under the selfconsistent-field conditions. We assume in our derivations arbitrary distribution functions and correlation properties of injected electrons in order to generalize the model to the practically important cases of nanoscale devices with nonequilibrium electron injection, like in a resonant-tunnelingdiode emitter, superlattice emitter, hot-electron emitter, etc. (see, e.g., Refs. 23-26). The particular case of a threedimensional (3D) Fermi-Dirac injection has also been addressed. The obtained results clearly demonstrate that both the current and voltage noise can be substantially reduced owing to the long-range Coulomb interactions. This result is very encouraging from the point of view of applications.

The paper is organized as follows. In Sec. II we introduce the basic equations describing the space-charge-limited (SCL) ballistic transport. In Sec. III the self-consistent steady-state spatial profiles for the electron density, electric field, and potential are found for an arbitrary injection distribution. The mean current and conductance are obtained in Sec. IV. Section V describes a general formula which relates both current and voltage fluctuations with the fluctuations of the occupation numbers in the leads. The current noise power, suppressed by interactions, is compared in Sec. VI with the case when interactions are disregarded. The Coulomb and Fermi noise-suppression factors are discussed in Secs. VII and VIII, respectively, whereas the noise temperature is given in Sec. IX. The voltage noise power under a fixed-current conditions is derived in Sec. X. The implementation of the results for a GaAs ballistic conductor is presented in Sec. XI. Finally, Sec. XII summarizes the main contributions of the paper, whereas in the Appendixes we present mathematical details concerning some derivations.

\section{BASIC EQUATIONS}

We consider a semiconductor ballistic sample attached to plane-parallel leads (Fig. 1). In a semiclassical framework, the electron occupation numbers $\widetilde{f}(x, \mathbf{k}, t)$ inside the ballistic conductor are determined by the electron flows from the left and right leads. The distribution of carriers is nonhomogeneous along the conductor: their concentration is higher near the leads and lower in the middle of the sample. The inhomogeneity of the space charge disturbs the electrostatic potential in such a way that the self-consistent built-in field 
determines the potential barrier, at which electrons are either reflected or transmitted depending on their energy (Fig. 1). We neglect tunneling and quantum reflection, i.e., the transmission probability is 1 if the electron energy is higher than the barrier height, and it is 0 in the opposite case. Out of equilibrium, the barrier height is different for the left and right lead electrons. If for the left electrons the barrier height is $\Phi_{b}$, for the right electrons the barrier height is $\Phi_{b}+q U$ (Fig. 1). This leads to an asymmetry in their contribution to the current: as the bias is increased, the barrier for the left electrons progressively decreases and the current from the left lead enhances, whereas the barrier for the right electrons increases and the current from the right lead decreases disappearing at all at high biases.

The occupation numbers are described by the Vlasov equation (collisionless Boltzmann equation with a selfconsistent field) $)^{18,27}$

$$
\left(\frac{\partial}{\partial t}+\frac{\hbar k_{x}}{m} \frac{\partial}{\partial x}+q \frac{d \tilde{\varphi}}{d x} \frac{\partial}{\hbar \partial k_{x}}\right) \tilde{f}(x, \mathbf{k}, t)=0,
$$

where $m$ is the electron effective mass, $\mathbf{k}=\left(k_{x}, \mathbf{k}_{\perp}\right)$, and $\tilde{\varphi}(x, t)$ is the self-consistent electric potential determined by the Poisson equation

$$
\frac{\partial^{2} \tilde{\varphi}}{\partial x^{2}}=\frac{q}{\kappa} \int \frac{d \mathbf{k}}{(2 \pi)^{d}} \tilde{f}(x, \mathbf{k}, t) .
$$

Here $\kappa$ is the dielectric permittivity and $d$ is the dimension of a momentum space (the spin variable is neglected). Since carriers move without collisions, the only source of noise arises from the random injection of carriers from the leads. Thus the boundary conditions at the left $(L)$ and right $(R)$ leads are:

$$
\begin{gathered}
\left.\widetilde{f}(0, \mathbf{k}, t)\right|_{k_{x}>0}=f_{L}(\mathbf{k})+\delta f_{L}(\mathbf{k}, t), \\
\left.\widetilde{f}(l, \mathbf{k}, t)\right|_{k_{x}<0}=f_{R}(\mathbf{k})+\delta f_{R}(\mathbf{k}, t), \\
\tilde{\varphi}(l, t)-\tilde{\varphi}(0, t)=\widetilde{U}(t),
\end{gathered}
$$

where $\delta f_{L, R}$ are the stochastic forces inside the leads with zero average and given correlation properties, and $\widetilde{U}$ is the applied bias between $x=0$ and $x=l$ (the potential drop inside the leads is neglected). As a consequence of stochastic injection, the occupation numbers $\widetilde{f}(x, \mathbf{k}, t)=f(x, \mathbf{k})$ $+\delta f(x, \mathbf{k}, t)$ and hence the potential $\tilde{\varphi}(x, t)=\varphi(x)$ $+\delta \varphi(x, t)$ fluctuate in time around their time-averaged values.

The leads are assumed to be completely absorptive, and the transverse electron momenta are conserved. Thus, one can make summing up over the transversal states (the summation can be replaced by integration due to the assumption of a large number of modes) and introduce for each longitudinal energy $\varepsilon$ the (fluctuating) occupation factor at a cross section $x$ :

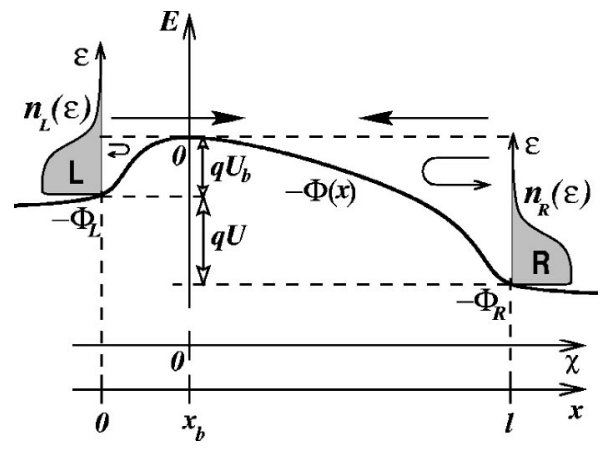

FIG. 2. Potential-energy profile for a ballistic space-chargelimited conductor. Electrons with energies $E>0$ pass over the barrier, while those with $E<0$ are reflected back to the leads. Shadowed regions illustrate the energy distributions of the occupation factors at the leads.

$$
\tilde{n}(x, \varepsilon, t)=\int_{0}^{\infty} \widetilde{f}\left(x, \varepsilon, \varepsilon_{\perp}, t\right) \nu_{\perp} d \varepsilon_{\perp},
$$

where $\varepsilon=\hbar^{2} k_{x}^{2} /(2 m), \varepsilon_{\perp}=\hbar^{2} \mathbf{k}_{\perp}^{2} /(2 m)$, and $\nu_{\perp}$ is the density of transverse modes ( $\nu_{\perp}=m / 2 \pi \hbar^{2}$ for the $3 \mathrm{D}$ case). The number of occupied transversal modes is $N_{\perp}=n A$, where $A$ is the cross-sectional area. In the semiclassical description applied here for a thick conductor, the number of the occupied transversal modes is assumed to be large, $N_{\perp} \gg 1$.

\section{SELF-CONSISTENT STEADY-STATE SPATIAL PROFILES}

It is advantageous to introduce the mean total longitudinal energy $E$ as a sum of the kinetic energy $\varepsilon$ and the potential energy $[-\Phi(x)]$ :

$$
E=\varepsilon-\Phi(x)
$$

We shall count off the potential energy from the barrier top: $\Phi(x) \equiv q \varphi(x)-q \varphi\left(x_{b}\right)$. Therefore, at the barrier position $x$ $=x_{b}$, we obtain $E=0$ for electrons having the injection kinetic energy equal to the barrier height (for both leads). The boundary values for the potential energy are expressed through the barrier height $q U_{b}$ and the applied bias $U$ as $\Phi_{L} \equiv \Phi(0)=q U_{b}$, and $\Phi_{R} \equiv \Phi(l)=\Phi_{L}+q U$ (Fig. 2). The solution of Eq. (4) for the stationary case $(\partial / \partial t=0)$ gives, after integration over the transversal states, the electron density at any section of the conductor in terms of the potential $\Phi,{ }^{18,27}$

$$
\begin{aligned}
N(\Phi)= & \int_{0}^{\infty}\left[n_{L}\left(E+\Phi_{L}\right)+n_{R}\left(E+\Phi_{R}\right)\right] \nu(E+\Phi) d E \\
& +2 \int_{-\Phi}^{0}\left[\theta(-\chi) n_{L}\left(E+\Phi_{L}\right)\right. \\
& \left.+\theta(\chi) n_{R}\left(E+\Phi_{R}\right)\right] \nu(E+\Phi) d E,
\end{aligned}
$$

where $n_{L, R}(E)=\int_{0}^{\infty} f_{L, R}\left(E, \varepsilon_{\perp}\right) \nu_{\perp} d \varepsilon_{\perp}$ are the occupation factors at the leads, $\nu(E)=1 /[2 \pi \hbar v(E)]$ the density of states, and $v=\sqrt{2 E / m}$ the velocity. The first integral in Eq. (9) corresponds to the electrons transmitted over the barrier 
( $E>0)$, while the second integral is referred to the reflected carriers $(-\Phi<E<0)$. The latter term is doubled, since for each energy there are two states with opposite momentum: $k_{x}$ and $-k_{x}$. Finally, $\theta(\chi)$ with $\chi=x-x_{b}$ is the Heaviside function that distinguishes two classes of the reflected carriers: those at the left of the barrier $(\chi<0)$ originated from the left lead, and those at the right $(\chi>0)$ coming from the right lead.

The electron density [Eq. (9)] can now be substituted into the Poisson equation $d^{2} \Phi / d x^{2}=\left(q^{2} / \kappa\right) N(\Phi)$ to find the self-consistent potential $\Phi$. The first integration gives the electric-field distribution

$$
\mathcal{E}(\Phi)=-\frac{1}{q} \frac{d \Phi}{d x}=-\operatorname{sgn}(\chi) \sqrt{\frac{2}{\kappa}} \sqrt{h(\Phi)},
$$

where

$$
\begin{aligned}
h(\Phi)=\int_{0}^{\Phi} N(\widetilde{\Phi}) d \widetilde{\Phi}= & \frac{m}{2 \pi \hbar}\left\{\int_{0}^{\infty}\left[n_{L}\left(E+\Phi_{L}\right)+n_{R}\left(E+\Phi_{R}\right)\right][v(E+\Phi)-v(E)] d E\right. \\
& \left.+2 \int_{-\Phi}^{0}\left[\theta(-\chi) n_{L}\left(E+\Phi_{L}\right)+\theta(\chi) n_{R}\left(E+\Phi_{R}\right)\right] v(E+\Phi) d E\right\} .
\end{aligned}
$$

Integrating Eq. (10), one obtains the distribution of the potential for both regions $\chi<0$ and $\chi>0$ in an implicit form

$$
q \sqrt{\frac{2}{\kappa}} \chi=-\operatorname{sgn}(\chi) \int_{0}^{\Phi} \frac{d \widetilde{\Phi}}{\sqrt{h(\widetilde{\Phi})}} .
$$

Matching the two branches at $\chi=0$ yields

$$
q l \sqrt{\frac{2}{\kappa}}=\int_{0}^{\Phi_{L}} \frac{d \Phi}{\sqrt{h_{-}(\Phi)}}+\int_{0}^{\Phi_{R}} \frac{d \Phi}{\sqrt{h_{+}(\Phi)}},
$$

with $h_{-} \equiv h(\chi<0)$ and $h_{+} \equiv h(\chi>0)$. Equation (13) relates three important parameters: the self-consistent barrier height $U_{b}$, the applied bias $U$, and the length of the conductor $l$. Given any two of them, the third one can be calculated ${ }^{28}$ from Eq. (13). In Ref. 18 a similar expression was obtained for the Maxwell-Boltzmann injection distribution. Here we have generalized it to the case of an arbitrary injection distribution profiles $n_{L}(E)$ and $n_{R}(E)$ at the leads. It should be noted that in Eq. (13) the dependence on bias enters not only through the upper limits of the integrals, but also through the functions $h_{ \pm}$.

If we assume that electrons inside the leads obey the equilibrium Fermi-Dirac (FD) distributions (the usual assumption in other works) from Eq. (7) one obtains the occupation numbers

$$
n_{L, R}(\varepsilon)=\frac{m k_{B} T}{2 \pi \hbar^{2}} \ln \left\{1+\exp \left[\left(\varepsilon_{F}-\varepsilon\right) / k_{B} T\right]\right\},
$$

where $\varepsilon_{F}$ is the Fermi energy at the lead and $T$ is the temperature. Thus, for the FD case, the steady-state spatial profiles of the potential, electric field, and electron density are determined through Eqs. (9)-(13) by making use of the distributions

$$
n_{L}\left(E+\Phi_{L}\right)=\frac{\mathcal{N}}{\xi A} \mathcal{F}_{0}\left(\alpha-E / k_{B} T\right)
$$

$$
n_{R}\left(E+\Phi_{R}\right)=\frac{\mathcal{N}}{\xi A} \mathcal{F}_{0}\left[\alpha-(E+q U) / k_{B} T\right],
$$

where $\mathcal{N}=\left(k_{F}^{2} A / 4 \pi\right)$ is the number of transversal modes in the degenerate zero-temperature limit, $\xi=\varepsilon_{F} / k_{B} T$ is the reduced Fermi energy, $\alpha=\left(\varepsilon_{F}-\Phi_{L}\right) / k_{B} T$ is the parameter characterizing the position of the Fermi energy with respect to the potential barrier, and $\mathcal{F}_{k}$ is the Fermi-Dirac integral of index $k^{27}$

\section{MEAN CURRENT AND CONDUCTANCE}

The mean ballistic current is found as an integral over the occupation numbers for the transmitted $(E>0)$ carriers from both leads:

$$
I=\frac{q A}{2 \pi \hbar} \int_{0}^{\infty}\left[n_{L}\left(E+\Phi_{L}\right)-n_{R}\left(E+\Phi_{R}\right)\right] d E .
$$

It is convenient for future analysis to introduce the energyresolved injection currents

$$
I_{L, R}(E)=\frac{q A}{2 \pi \hbar} n_{L, R}(E) .
$$

By this definition, the mean current is

$$
I=\int_{0}^{\infty}\left[I_{L}\left(E+\Phi_{L}\right)-I_{R}\left(E+\Phi_{R}\right)\right] d E .
$$

Having found the barrier height $\Phi_{L}$ from Eq. (13), this equation determines the current-voltage characteristics. Since we have not assumed that the bias must be small, this characteristics is nonlinear in bias in a general case.

For the FD case, Eq. (18) reduces to

$$
I=\frac{q}{2 \pi \hbar} \mathcal{N} \frac{k_{B} T}{\xi}\left[\mathcal{F}_{1}(\alpha)-\mathcal{F}_{1}(\alpha-V)\right],
$$

where we have denoted the dimensionless bias $V$ $=q U /\left(k_{B} T\right)$. It is seen that under the ballistic SCL conduction, the current is determined by the relative positions of the 
Fermi energies at the leads and the barrier top through theparameters $\alpha$ and $V$. This is in contrast to the case of diffusive conductors, in which the current is determined by the scattering strength.

The differential conductance $G=d I / d U$ is obtained from Eq. (16) as

$$
G=\frac{q^{2}}{2 \pi \hbar} A\left\{n_{R}\left(\Phi_{R}\right)-\frac{d U_{b}}{d U}\left[n_{L}\left(\Phi_{L}\right)-n_{R}\left(\Phi_{R}\right)\right]\right\} .
$$

The derivative $d U_{b} / d U$ is calculated in Appendix A. With its help the formula for the conductance becomes

$$
G=\frac{q^{2}}{2 \pi \hbar} A\left[n_{L}\left(\Phi_{L}\right) \frac{\Delta_{R}}{\Delta}+n_{R}\left(\Phi_{R}\right) \frac{\Delta_{L}}{\Delta}\right] .
$$

It is seen that the conductance is a sum of two contributions corresponding to the left and right leads. Each of them is a product of the conductance unit $G_{0}=q^{2} /(2 \pi \hbar)$, the number of the transversal modes for the injection energy corresponding to the barrier top, and some Coulomb interaction factors determined through $\Delta_{L, R}$ given in Appendix A. These factors depend on the whole electron system and cannot be separated for the left and right lead electrons.

At small biases close to equilibrium, by assuming identical leads (e.g., FD distributions), we obtain $\Delta_{L, R} \approx \Delta / 2$. For this case the interaction factors vanish, and the conductance reduces to the value given by the multichannel Landauer formula

$$
G_{e q} \approx \frac{q^{2}}{2 \pi \hbar} N_{\perp}\left(\Phi_{L}^{0}\right)
$$

where $N_{\perp}\left(\Phi_{L}^{0}\right)=A n_{L}\left(\Phi_{L}^{0}\right)$ is the number of open modes at the barrier energy. Under this small-bias condition, the current-voltage characteristic is linear: $I \approx G_{e q} U$. Equation (21) may be viewed as the extension of the Landauer formula for the conductance to far-from-equilibrium conditions for interacting electrons in a SCL ballistic conductor.

In the opposite limit of high biases $U_{b} \ll U<U_{c r}$, where $U_{c r}$ is the critical bias under which the barrier vanishes, the asymptotic formula for the current is ${ }^{27}$

$$
I_{A} \approx I_{\text {Child }}\left[1+\frac{3}{\sqrt{\Phi_{R}}} \frac{\int_{0}^{\infty} I_{L}\left(E+\Phi_{L}\right) \sqrt{E} d E}{\int_{0}^{\infty} I_{L}\left(E+\Phi_{L}\right) d E}\right],
$$

where the leading-order term is the Child current

$$
I_{\text {Child }}=\frac{4}{9} \kappa A \sqrt{\frac{2}{m}} \frac{\Phi_{R}^{3 / 2}}{q l^{2}} .
$$

The main term $\propto U^{3 / 2}$ is independent of the injection, while the second-order term $\propto U$ contains information on the injection occupation numbers. Equation (23) for the FD case was presented in Ref. 29.
The asymptotic behavior of the conductance at high biases is obtained from Eq. (23)

$$
G_{A}=\frac{3}{2} \frac{q I_{\text {Child }}}{\Phi_{R}}\left[1+\frac{2}{\sqrt{\Phi_{R}}} \frac{\int_{0}^{\infty} I_{L}\left(E+\Phi_{L}\right) \sqrt{E} d E}{\int_{0}^{\infty} I_{L}\left(E+\Phi_{L}\right) d E}\right],
$$

giving the leading-order term $G_{A} \approx(3 / 2)\left(I_{\text {Child }} / U\right) \sim \sqrt{U}$ for an arbitrary injection. For the FD case, Eq. (25) leads to

$$
G_{A}=\frac{3}{2} \frac{I_{\text {Child }}}{U}\left[1+\sqrt{\frac{\pi k_{B} T}{q U}} \frac{\mathcal{F}_{3 / 2}(\alpha)}{\mathcal{F}_{1}(\alpha)}\right],
$$

\section{SELF-CONSISTENT CURRENT AND VOLTAGE FLUCTUATIONS. GENERAL FORMULAS}

According to the definition of the potential energy in Sec. III, the potential fluctuations at any point $x$ are given by $\delta \Phi_{x}=q \delta \varphi(x)-q \delta \varphi\left(x_{b}\right)$. In the nonstationary frame fixed to the barrier top, the fluctuations at the barrier position are zero, $\delta \Phi_{x_{b}}=0$, whereas at the leads they are: $\delta \Phi_{0} \equiv \delta \Phi_{L}$ and $\delta \Phi_{l} \equiv \delta \Phi_{R}=\delta \Phi_{L}+q \delta U$, where $\delta U$ is the fluctuation of the applied bias.

The current fluctuation is obtained by integrating over the energy the fluctuation of the occupation factor $\delta n(E)$ found from a linearization of Eq. (4) around the mean values. ${ }^{27}$ One obtains

$$
\begin{aligned}
\delta I= & \int_{0}^{\infty}\left[\delta I_{L}\left(E+\Phi_{L}\right)-\delta I_{R}\left(E+\Phi_{R}\right)\right] d E \\
& -I_{L}\left(\Phi_{L}\right) \delta \Phi_{L}+I_{R}\left(\Phi_{R}\right) \delta \Phi_{R} .
\end{aligned}
$$

where $\delta I_{L, R}(E)$ are the energy-resolved injection-current fluctuations from each lead. In Eq. (27), only the lowfrequency current fluctuations are considered, i.e., the frequencies are below the inverse electron transit time between the leads and the displacement current is neglected. The first integral term is standard, and corresponds to the injected current fluctuation $\delta I_{i n j}$. The last two terms are the fluctuations induced by the self-consistent potential fluctuations, that give rise to the long-range Coulomb correlations. ${ }^{27}$ To find those terms, we need to obtain $\delta \Phi_{L}$ or, equivalently, the self-consistent fluctuations of the barrier height in terms of the injected fluctuations $\delta I_{L, R}$ by solving the Poisson equation. This has been done in the Appendix B. The result is the relation

$$
\begin{aligned}
\delta I-G \delta U= & \int_{-\Phi_{L}}^{\infty} \gamma_{L}(E) \delta I_{L}\left(E+\Phi_{L}\right) d E \\
& +\int_{-\Phi_{R}}^{\infty} \gamma_{R}(E) \delta I_{R}\left(E+\Phi_{R}\right) d E,
\end{aligned}
$$

where $G$ is the differential conductance [Eq. (21)], and the functions $\gamma_{L, R}(E)$ are determined by 


$$
\begin{aligned}
& \gamma_{L}(E)=\left\{\begin{array}{l}
-2 C_{\Delta} \int_{-E}^{\Phi_{L} v(E+\Phi)} \frac{h_{-}^{3 / 2}}{h^{2}} d \Phi, \quad-\Phi_{L}<E<0 \\
1-C_{\Delta}\left[\int_{0}^{\Phi_{L} v(E+\Phi)-v(E)} \frac{\int_{-}^{3 / 2}}{h^{3 / 2}} d \Phi+\int_{0}^{\Phi_{R} v(E+\Phi)-v(E)} \frac{h_{+}^{3 / 2}}{h^{2}} d \Phi\right], \quad 0<E<\infty,
\end{array}\right. \\
& \gamma_{R}(E)=\left\{\begin{array}{l}
-2 C_{\Delta} \int_{-E}^{\Phi_{R} v(E+\Phi)} \frac{h_{+}^{3 / 2}}{h_{+}} d \Phi, \quad-\Phi_{R}<E<0 \\
-1-C_{\Delta}\left[\int_{0}^{\Phi_{L} v(E+\Phi)-v(E)} \frac{\int_{-}^{3 / 2}}{h^{3 / 2}} d \Phi+\int_{0}^{\Phi_{R} v(E+\Phi)-v(E)} \frac{h_{+}^{3 / 2}}{h^{3 / 2}}\right], \quad 0<E<\infty .
\end{array}\right.
\end{aligned}
$$

In Eq. (29), one can distinguish the contributions from the left-lead $\left(\gamma_{L}\right)$ and right-lead $\left(\gamma_{R}\right)$ electrons, as well as from the reflected $(E<0)$ and transmitted $(E>0)$ carriers. All the terms related to the barrier fluctuations are proportional to the constant

$$
C_{\Delta}=\frac{m}{2 \pi \hbar \Delta}\left[n_{L}\left(\Phi_{L}\right)-n_{R}\left(\Phi_{R}\right)\right],
$$

where $\Delta$ is the constant previously used to determine the conductance $G$ and which has been derived in Appendix A. Equations (28)-(30) are one of the main results of our theory. They relate the self-consistent current and voltage fluctuations with the noise source-spontaneous fluctuations of the occupation numbers in the leads. The transfer functions $\gamma_{L, R}$, summarizing the interaction effects, show the contribution of each energy to the total fluctuations. In the absence of interactions, $\gamma_{L}(E)=\theta(E)$ and $\gamma_{R}(E)=-\theta(E)$, i.e., the fluctuations of all energies above the barrier top are equally transmitted. The role of the Coulomb interactions is to introduce an inhomogeneity in the energy flux of fluctuations, by suppressing or enhancing occupation-number fluctuations at different energies. Note that the Coulomb interactions are pronounced only in the presence of transport. In equilibrium, $C_{\Delta}=0$, and they are not effective.

In general, both terms $\gamma_{L}$ and $\gamma_{R}$ may contribute to the noise. However, at high biases, $U_{b} \ll U<U_{c r}$, one can find that only $\gamma_{L}(E>0)$ dominates, the asymptotic expression for which is given by ${ }^{27}$

$$
\begin{gathered}
\gamma_{L}^{A}(E)=\frac{3}{\sqrt{\Phi_{R}}}\left(\sqrt{E}-v_{\Delta}\right)+O\left(\frac{1}{\Phi_{R}}\right), \\
v_{\Delta}=\frac{1}{n_{L}\left(\Phi_{L}\right)} \int_{0}^{\infty}\left[-\frac{\partial n_{L}\left(E+\Phi_{L}\right)}{\partial E}\right] \sqrt{E} d E .
\end{gathered}
$$

We shall use these formulas later on to analyze the asymptotic limits for other important noise quantities.

To find the total fluctuations $\delta I$ or $\delta U$, one needs to define the correlation properties of the fluctuations at the leads. In general, one can write ${ }^{27}$

$$
\left\langle\delta I_{k}(E) \delta I_{k}\left(E^{\prime}\right)\right\rangle=K_{k}(E)(\Delta f) \delta\left(E-E^{\prime}\right),
$$

where, $k=L, R$ and $\Delta f$ is the frequency bandwidth (we assume the low-frequency limit). For the case of the Poissonian injection from both leads $K_{L, R}(E) \propto I_{L, R}(E)$. More generally, for the non-Poissonian injection, under the assumption that the leads are in local equilibrium, one can use the formula

$$
K_{L, R}(E)=2 k_{B} T G_{0} A\left(-\frac{\partial n_{L, R}}{\partial E}\right)
$$

where $G_{0}$ is the unit of conductance. This formula follows from the Nyquist theorem (see Appendix C).

By applying Eq. (34) for the FD case, we also obtain

$$
K_{L, R}(E)=\frac{2 G_{S}}{\xi} \frac{1}{1+e^{\left(E / k_{B} T\right)-\xi}},
$$

where $G_{S}=G_{0} \mathcal{N}$ is the Sharvin conductance, and $G_{0}$ $=q^{2} /(2 \pi \hbar)$ is the unit of conductance. For further noise analysis, we have to specify the conditions imposed on the external circuit. We shall consider two cases of interest: (i) a voltage-controlled circuit (zero external impedance) for which $\delta U=0$, and one can find the spectral density of current fluctuations $S_{I}$; and (ii) a current controlled circuit (infinite external impedance) for which $\delta I=0$ and one can find the spectral density of voltage fluctuations $S_{V}$ on the leads. In both cases, we will show that Coulomb interactions play a prominent role in the noise suppression.

\section{COULOMB SUPPRESSED CURRENT NOISE}

Let us suppose that the potentials at the leads are held fixed and do not fluctuate. This corresponds to the case when currents are measured using a zero-impedance external circuit. Under the condition $\delta U=0$, Eq. (28) gives the currentnoise spectral density

$$
\begin{aligned}
S_{I}= & \int_{-\Phi_{L}}^{\infty} \gamma_{L}^{2}(E) K_{L}\left(E+\Phi_{L}\right) d E \\
& +\int_{-\Phi_{R}}^{\infty} \gamma_{R}^{2}(E) K_{R}\left(E+\Phi_{R}\right) d E
\end{aligned}
$$


It is important to highlight that the obtained current-noise power [Eq. (36)], that includes Coulomb interactions, has been obtained for a wide range of biases, ranging from equilibrium to far-from-equilibrium conditions beyond the linearresponse regime. Therefore, it describes both thermal and shot-noise limits.

One can verify that Eq. (36) at the high-bias limit $U_{b}$ $\ll U<U_{\text {cr }}$ reduces to

$$
S_{I}^{A}=\int_{0}^{\infty} \gamma_{L}^{2}(E) K_{L}\left(E+\Phi_{L}\right) d E
$$

Taking into account Eqs. (31) and (34), one obtains the asymptotic expression for the current-noise power

$$
S_{I}^{A} \approx \beta 2 q I \frac{k_{B} T}{q U}=\frac{\beta}{3} 4 k_{B} T G_{A},
$$

where

$$
\beta=9\left(1-\frac{\left[\int_{0}^{\infty} I_{L}\left(E+\Phi_{L}\right) \frac{d E}{2 \sqrt{E}}\right]^{2}}{I_{L}\left(\Phi_{L}\right) \int_{0}^{\infty} I_{L}\left(E+\Phi_{L}\right) d E}\right) .
$$

The parameter $\beta$ is determined by the energy profile of the injected electrons $I_{L}(E)$. For the FD injection, Eq. (39) leads to the formula derived earlier ${ }^{29}$ :

$$
\beta(\alpha)=9\left(1-\frac{\pi}{4} \frac{\left[\mathcal{F}_{1 / 2}(\alpha)\right]^{2}}{\mathcal{F}_{0}(\alpha) \mathcal{F}_{1}(\alpha)}\right) .
$$

Equation (39) is more general and can be applied to an arbitrary injection distribution obeying the Nyquist relationship [Eq. (34)] for the correlation function. It is seen also from Eq. (38), that at high biases $S_{I}^{A} \sim \sqrt{U}$.

One can also find, for comparison, the current-noise power for the case of disregarded Coulomb correlations

$$
S_{I}^{\text {uncor }}=\int_{0}^{\infty} K_{L}\left(E+\Phi_{L}\right) d E+\int_{0}^{\infty} K_{R}\left(E+\Phi_{R}\right) d E
$$

which under the assumption of equilibrium conditions at the leads [Eq. (34)] results in

$$
S_{I}^{\mathrm{uncor}}=2 q k_{B} T\left[I_{L}\left(\Phi_{L}\right)+I_{R}\left(\Phi_{R}\right)\right]
$$

For the sake of completeness, we present also expression for the FD case:

$$
S_{I}^{\text {uncor }}=2 k_{B} T G_{S} \frac{1}{\xi}\left[\mathcal{F}_{0}(\alpha)+\mathcal{F}_{0}(\alpha-V)\right] .
$$

Note that Eq. (42) corresponds to Eq. (3) discussed in Sec. I. Indeed, if one applies Eq. (3) for two opposite flows of noninteracting FD electrons, summing up over the open channels, one then gets Eq. (42).

\section{COULOMB NOISE-SUPPRESSION FACTOR}

To estimate the significance of Coulomb interactions, one can introduce the Coulomb noise-suppression factor ${ }^{29}$

$$
\Gamma_{C}=\frac{S_{I}}{S_{I}^{\text {uncor }}}
$$

that extends over both thermal-noise and shot-noise limits. Strictly in equilibrium, $\Gamma_{C}=1$, as was pointed out in Sec. V. The effect of interactions is noticeable, however, already under small applied biases. In Sec. XI A, we will show that while $S_{I}^{\text {uncor }}$ increases with bias, the behavior of $S_{I}$ is just the opposite: it decreases with bias starting from $U=0$ up to a certain bias where it reaches the noise minimum, then $S_{I}$ increases but much slower than $S_{I}^{\text {uncor }}$.

We remark the difference between the noise-suppression factor [Eq. (43)] and the shot-noise-suppression factor (also referred to as the Fano factor ${ }^{3}$ ) given by

$$
F=\frac{S_{I}^{e x}}{2 q I}
$$

In the latter formula, the noise power $S_{I}^{e x}$ refers to the shotnoise power, i.e., the excess to the thermal-equilibrium-noise level: ${ }^{6} S_{I}^{e x}=S_{I}-4 k_{B} T G_{e q}$. Moreover, Eq. (44) is meaningful for systems in which $S_{I}^{e x} \propto I$ (for instance, in linearresponse regime). In this case, it simply gives a measure of how much the noise power deviates from the ideal Poissonian $2 q I$ value due to correlations among carriers. For the nonlinear case, when $S_{I}^{e x}$ is not proportional to $I$, definition (44) is less useful, since the suppression factor depends on $I$. It should be noted that Eqs. (43) and (44) become identical under the conditions: $q U \gg k_{B} T$ (for negligible thermal-noise contribution) and $S_{I}^{\text {uncor }}=2 q I$. The latter is valid, for instance, for the Maxwell-Boltzmann nondegenerate injection. ${ }^{18}$ If the injection is non-Poissonian, as in the case of FD injection, $S_{I}^{\text {uncor }} \neq 2 q I$, and Eqs. (43)and (44) differ.

\section{FERMI NOISE-SUPPRESSION FACTOR}

It is instructive to introduce the Poissonian noise power for the full range of biases:

$$
\begin{aligned}
S_{I}^{P} & =2 q I \operatorname{coth}\left(\frac{q U}{2 k_{B} T}\right) \\
& \approx\left\{\begin{array}{cc}
4 k_{B} T G_{e q}, & q U \ll k_{B} T \\
2 q I, & q U \gg k_{B} T .
\end{array}\right.
\end{aligned}
$$

Based on this definition, one can introduce the Fermi noisesuppression factor

$$
\Gamma_{F}=\frac{S_{I}^{\text {uncor }}}{S_{I}^{P}}
$$

Thus the total noise-suppression factor is 


$$
\Gamma=\Gamma_{F} \Gamma_{C}=\frac{S_{I}}{S_{I}^{P}},
$$

Note that all of definitions (43), (46), and (47) extend from thermal- to shot-noise limits. They will be used in the analysis of the results in Sec. XI.

For the FD two-lead injection, from Eqs. (19) and (42) it follows that

$$
\Gamma_{F}=\frac{\mathcal{F}_{0}(\alpha)+\mathcal{F}_{0}(\alpha-V)}{\mathcal{F}_{1}(\alpha)-\mathcal{F}_{1}(\alpha-V)} \tanh (V / 2) .
$$

One can verify that at the low-bias limit $V \rightarrow 0$, there is no suppression effect: $\Gamma_{F} \rightarrow 1$. The finite bias introduces asymmetry in the contributions from electrons of different leads. The larger the bias, the smaller is the contribution from the biased lead, since the electrons have a higher potential barrier by an additional amount of $V$ to overcome. It is clear that starting from a certain bias, the contribution from only one injecting lead dominates. The unidirectional charge flow occurs when

$$
V \gtrsim \max \{5 ; \alpha+3\} .
$$

This condition comes from the consideration of two limits. For a nondegenerate injection (the Fermi energy is below the barrier, $\alpha \lesssim-3$ ) the bias-to-temperature ratio should be large: $q U \gtrsim 5 k_{B} T$, whereas for a highly degenerate injection (the Fermi energy is above the barrier, $\alpha \gtrsim 3$ ), the bias should be compared with the Fermi energy: $q U \gtrsim \varepsilon_{F}-q U_{b}$ $+3 k_{B} T$. Thus, under condition (49), from Eq. (48) one obtains the asymptotic formula ${ }^{29}$

$$
\Gamma_{F} \approx \frac{\mathcal{F}_{0}(\alpha)}{\mathcal{F}_{1}(\alpha)} \approx\left\{\begin{array}{cc}
1, & \alpha \lesssim-3 \\
\frac{2}{\alpha+3 \pi / \alpha^{2}}, & \alpha \gtrsim 3 .
\end{array}\right.
$$

It is also of interest to analyze the case opposite to condition (49), when the bias is not so high that both leads contribute to the charge flow, namely, $V \leqq \max \{5 ; \alpha+3\}$. Then, for a nondegenerate limit $\Gamma_{F} \approx 1$. For a highly degenerate limit, that happens when $V \leqq \alpha-3$, one can use the approximations for the Fermi-Dirac integrals ${ }^{29} \mathcal{F}_{0}(x) \approx x$ and $\mathcal{F}_{1}(x) \approx x^{2} / 2+\pi^{2} / 6$, and Eq. (48) leads to a simple formula

$$
\Gamma_{F}=\frac{2}{V} \tanh \left(\frac{V}{2}\right) .
$$

Surprisingly, the dependence on $\alpha$ and hence on the barrier height, ballistic length, and material parameters, canceled out from this equation. $\Gamma_{F}$ depends only on one parameter-the bias-to-temperature ratio, and at sufficiently high $V$ it decreases with bias as $\Gamma_{F} \approx 2 / V$. Note that this behavior occurs under the nonlinear bias regime in the presence of a space charge. Indeed, for this case one finds the sublinear characteristics for the mean current $I=G_{S} U\left[1-q\left(U_{b}+U / 2\right) / \varepsilon_{F}\right]$, whereas the current noise power is given by $S_{I}^{\text {uncor }}$ $=4 k_{B} T G_{S}\left[1-q\left(U_{b}+U / 2\right) / \varepsilon_{F}\right]$, with the identical factor in square brackets. As a result, one obtains, $S_{I}^{\text {uncor }}=4 k_{B} T I / U$, as in the linear-response regime. It should be remembered, however, that at higher biases $V \gg \alpha$, the $2 / V$ dependence is changed to the $2 / \alpha$ law. The largest noise suppression by Fermi correlations ${ }^{29}$ is described by Eq. (50), giving $\Gamma_{F}^{\mathrm{min}}$ $=2 / \xi$, when the barrier height is zero. We shall give some examples in Sec. XI.

\section{NOISE TEMPERATURE}

It is interesting to see from Eq. (38), that at high biases and strong screening, despite the strong nonlinearity, the ratio between $S_{I}$ and the differential conductance $G$ tends to the constant value. It is instructive, therefore, to introduce the effective noise temperature $T_{n}$ through $^{30} S_{I}=4 k_{B} T_{n} G$. Note that $k_{B} T_{n}$ has a meaning of the maximum noise power per unit bandwidth which can be delivered to an output matched circuit; thus it is a measurable quantity. ${ }^{31}$ The asymptotic high-bias value is then obtained as

$$
\frac{T_{n}}{T}=\frac{1}{3} \beta(\alpha) \approx\left\{\begin{array}{cc}
3(1-\pi / 4), & \alpha \lesssim-3 \\
1 / 3, & \alpha \gg 1 .
\end{array}\right.
$$

It is seen that $T_{n}<T$ for any $\alpha$, indicating the noise suppression effect [see the plot of $\beta(\alpha)$ in Ref. 29]. For a nondegenerate Maxwell-Boltzmann injection, the limiting value $\left(T_{n}^{\text {ndeg }} / T\right)=3(1-\pi / 4) \approx 0.644$ is well known. ${ }^{30,32}$ For a highly degenerate FD injection, we have obtained from our theory $\left(T_{n}^{\mathrm{deg}} / T\right)=1 / 3$. The physical meaning of the latter result is that the noise power per unit bandwidth produced by the SCL ballistic conductor with degenerate FD electrons is $1 / 3$ of the thermal noise power produced by the heated resistance with the same value of the conductance $G$ (the same $I-V$ dependence), independently of the material parameters.

\section{COULOMB SUPPRESSED VOLTAGE NOISE}

Alternatively, one could measure the voltages at the leads using an ideal infinite-impedance voltmeter. The infiniteimpedance external circuit then forces the current to be zero at all times, $\delta I=0$. Fluctuations in the current are counterbalanced by fluctuations of the chemical potentials in the electron reservoirs. Under the condition $\delta I=0$, Eq. (28) gives the voltage-noise spectral density which takes into account the Coulomb correlations:

$$
\begin{aligned}
S_{U}= & \frac{1}{G^{2}}\left[\int_{-\Phi_{L}}^{\infty} \gamma_{L}^{2}(E) K_{L}\left(E+\Phi_{L}\right) d E\right. \\
& \left.+\int_{-\Phi_{R}}^{\infty} \gamma_{R}^{2}(E) K_{R}\left(E+\Phi_{R}\right) d E\right] .
\end{aligned}
$$

It is evident that the relation

$$
S_{U}=\frac{S_{I}}{G^{2}}
$$

holds, in which $S_{I}$ is the current-noise spectral density [Eq. (36)] measured under $\delta U=0$, and $G$ is the steady-state differential conductance (21). 


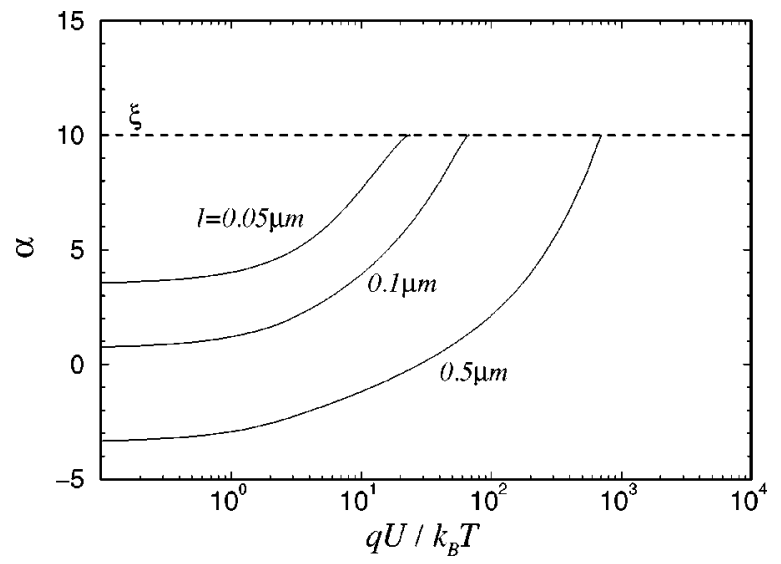

FIG. 3. Parameter $\alpha=\left(\varepsilon_{F}-\Phi_{L}\right) / k_{B} T$ characterizing the position of the Fermi energy $\varepsilon_{F}$ with respect to the potential barrier $\Phi_{L}$ for different lengths of the ballistic region $l$. At $U \rightarrow U_{c r}$ the barrier vanishes, $\Phi_{L}=0$, and $\alpha$ attains its maximum value $\alpha=\xi=10$.

The asymptotic behavior of $S_{U}$ at high biases can also be found from Eqs. (38) and (54). We obtain

$$
S_{U}^{A}=\frac{\beta}{3} 4 k_{B} T \frac{1}{G_{A}},
$$

with $\beta$ given by Eq. (39). It is seen that the voltage noise decreases with bias as $S_{U}^{A} \sim 1 / \sqrt{U}$ at $U_{b} \ll U<U_{c r}$ (the general result independent of the injection distribution). Hence the Coulomb interactions result in the voltage-noise suppression. This fact will be discussed in detail in Sec. XI B.

\section{RESULTS FOR FERMI-DIRAC INJECTION}

To illustrate our results, consider the GaAs ballistic $n-i-n$ diode at $T=4 \mathrm{~K} .{ }^{33}$ For this temperature and the effective mass $m=0.067 m_{0}$, the effective density of states is $N_{c}$ $\approx 6.7 \times 10^{14} \mathrm{~cm}^{-3}$. Assuming the contact doping 1.6 $\times 10^{16} \mathrm{~cm}^{-3}$, the reduced Fermi energy $\xi \approx 10$, and the contact electrons are degenerate, that is necessary for studying the joint effect of both Fermi and Coulomb correlations. For this set of parameters, the Debye screening length associated with the contact degenerate electron density is approximately $L_{D}=\sqrt{\kappa k_{B} T /\left[q^{2} N_{c} \mathcal{F}_{-1 / 2}(\xi)\right]} \approx 14 \mathrm{~nm}$. The calculations have been carried out for the following ballistic lengths: $l$ $=0.05,0.1$, and $0.5 \mu \mathrm{m}$.

The degeneracy of the contact electrons does not guarantee the degeneracy of the injection, since the potential barrier determines the energy portion of electrons which may pass over the barrier and contribute to the injection current. For each ballistic length $l$ and bias $U$, we have numerically solved Eq. (13) to find the self-consistent potential barrier height $\Phi_{L}$ and the parameter $\alpha=\left(\varepsilon_{F}-\Phi_{L}\right) / k_{B} T$ characterizing the position of the Fermi energy with respect to the potential barrier. The results are plotted in Fig. 3 .

It is seen that in equilibrium, for the ballistic lengths $l$ $=0.05,0.1$, and $0.5 \mu \mathrm{m}$, the self-consistent barrier height $\Phi_{L}$ is about $6 k_{B} T, 9 k_{B} T$, and $13 k_{B} T$, respectively. This means that the injected electrons at $U=0$ are degenerate for $l=0.05$ and $0.1 \mu \mathrm{m}$, and nondegenerate for $l=0.5 \mu \mathrm{m}$, since

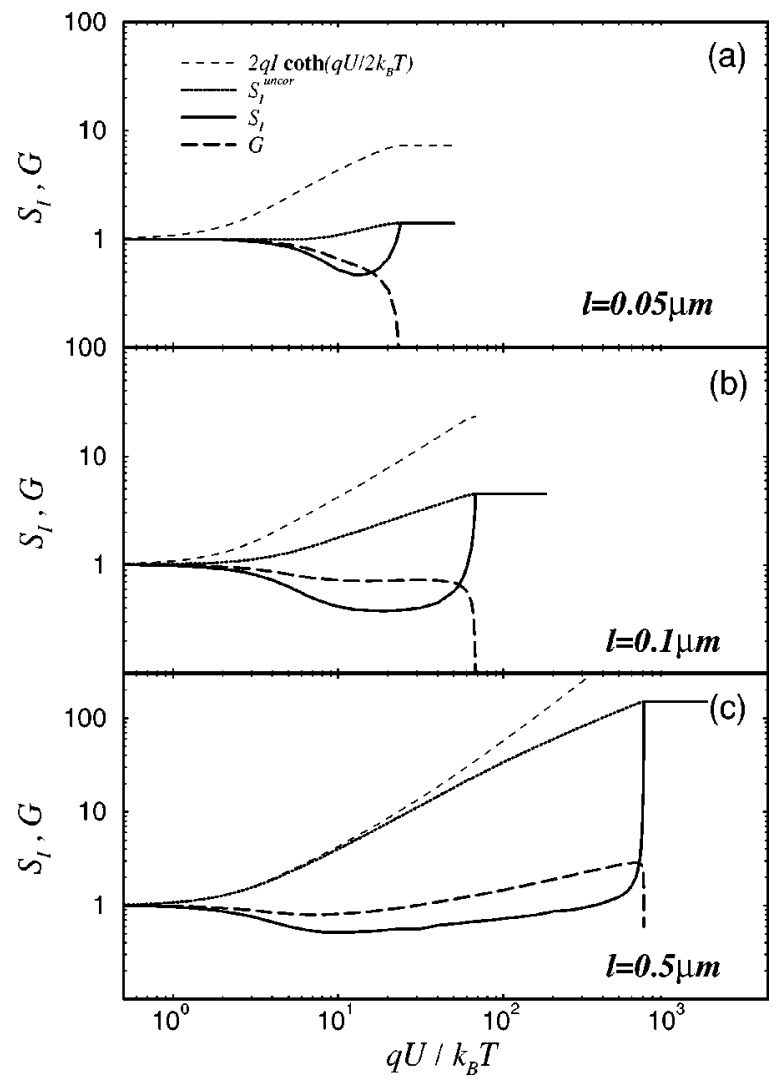

FIG. 4. Current-noise power $S_{I}$ and differential conductance $G$ vs bias $U$ for different ballistic lengths $l$. For comparison the noise power for the Poissonian injection $2 q I \operatorname{coth}\left(q U / 2 k_{B} T\right)$ and the Fermi-Dirac injection with disregarded Coulomb correlations $S_{I}^{\text {uncor }}$ are plotted. The normalization constants are the corresponding equilibrium values at $U=0: S_{I}^{e q}=4 k_{B} T G_{e q}$ and $G_{e q}$.

for the latter case only the tail of the distribution function is injected $(\alpha<-3)$. As $U$ is increased, $\Phi_{L}$ vanishes and the injection becomes degenerate for all three cases. Finally, at $U \rightarrow U_{c r}$ the potential barrier vanishes, $\alpha=\xi$, and the transport is no longer space-charge limited. The values of $U_{c r}$ depend obviously on the ballistic length $l$ (see Fig. 3).

\section{A. Fixed-bias conditions: $\delta V=0$}

In Fig. 4 we show the results for the current-noise power $S_{I}$ [Eq. (36)] and differential conductance $G$ vs bias $U$ calculated for different ballistic lengths $l$. For comparison, the noise power for the Poissonian injection $S_{I}^{P}$ [Eq. (45)] and Fermi-Dirac injection with disregarded Coulomb correlations $S_{I}^{\text {uncor }}$ [Eq. (42)] have also been plotted. In equilibrium, all the noise-power curves coalesce toward the Johnson-Nyquist noise $S_{I}^{e q}=4 k_{B} T G_{e q}$ independently of the presence of Fermi or Coulomb correlations. However, starting from small biases the difference becomes drastic. While $S_{I}^{\text {uncor }}$ increases with bias, the behavior of $S_{I}$ is just the opposite: it decreases with bias starting from $U=0$ up to a certain bias where it reaches the noise minimum, then $S_{I}$ increases, but much slower than $S_{I}^{\text {uncor }}$. Finally, at $U \rightarrow U_{c r}$ when the barrier vanishes, $S_{I}$ sharply recovers $S_{I}^{\text {uncor }}$. Note that in the absence of 


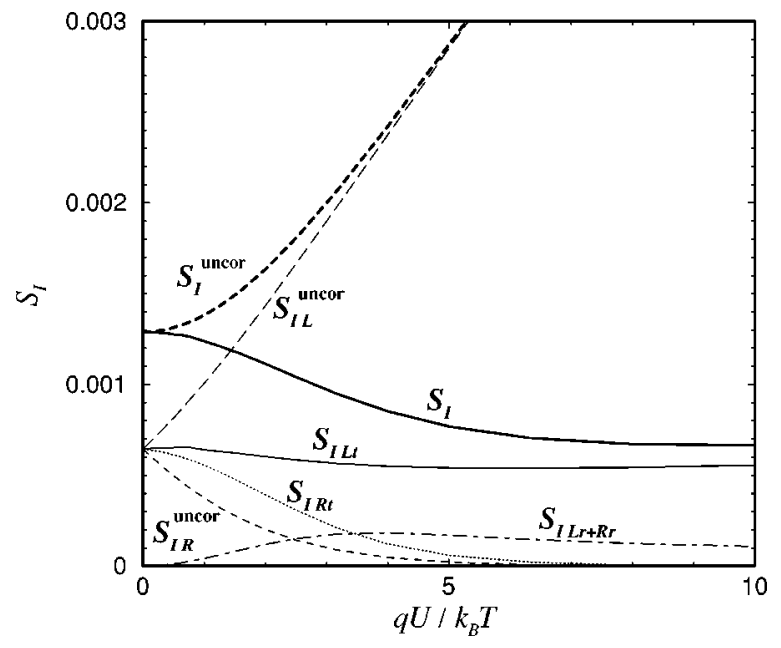

FIG. 5. Contributions to the current-noise power $S_{I}$ for the case of $l=0.5 \mu \mathrm{m}$ : from overbarrier electrons transmitted from the left $\left(S_{I L t}\right)$ and right $\left(S_{I R t}\right)$ leads, and those reflected by the barrier $\left(S_{I L r+R r}\right)$. For comparison, contributions to $S_{I}^{\text {uncor }}$ are shown as well: from left- $\left(S_{I L}^{\text {uncor }}\right)$ and right-lead $\left(S_{I R}^{\text {uncor }}\right)$ electrons.

Coulomb interactions, $S_{I}^{\text {uncor }}$ follows the Poissonian law $S_{I}^{P}$ $=2 q I \operatorname{coth}\left(q U / 2 k_{B} T\right)$ only for a nondegenerate injection, as in the case of $l=0.5 \mu \mathrm{m}$ at $q U \lesssim 10 k_{B} T$ [Fig. 4(c)]. At higher biases, and for shorter ballistic lengths in all the range, the injection is non-Maxwellian and $S_{I}<S_{I}^{P}$ because of Fermi suppression [Figs. 4(a) and 4(b)]. It should also be noted that $S_{I}<S_{I}^{e q}$ in a wide range of biases, which means that the noise for interacting ballistic electrons in an external field is less than the equilibrium Johnson-Nyquist noise. As will be shown later, the same is true for the voltage noise power.

Figure 5 assists the understanding of the results by showing the contributions to $S_{I}$ from different electron groups. The effect of Coulomb correlations is manifested quite differently for the left lead and right-lead electrons: while the left-lead noise is suppressed $\left(S_{I L t}<S_{I L}^{\text {uncor }}\right)$, the right-lead noise is enhanced $\left(S_{I R t}>S_{I R}^{\mathrm{uncor}}\right)$. Since the role of the rightlead electrons is diminished with bias, the overall effect of interaction results in the total-noise suppression. There is also a non-negligible contribution $(\sim 10-15 \%)$ to the noise from the reflected carriers at $q U \lesssim 10 k_{B} T$. It appears for correlated electrons only.

Figure 6 shows the noise temperature $T_{n}$ versus bias $U$ calculated from $\left(T_{n} / T\right)=S_{I} /\left(4 k_{B} T G\right)$ by using the data of Fig. 4. One can see that starting from $T_{n}=T$ at zero bias it drops at $q U \gtrsim k_{B} T$ below the temperature $T$ of the injected electrons. It is interesting to note that for degenerate electrons $(l=0.05 \mu \mathrm{m})$, this drop starts to appear at higher biases than for nondegenerate electrons $(l=0.5 \mu \mathrm{m})$. According to Eq. (52), the minimal asymptotic value of $T_{n}$ in the limit $l \rightarrow \infty, U \rightarrow \infty$ differs for nondegenerate and degenerate electrons (see indications in Fig. 6). For our set of parameters, the injection is degenerate at the highest biases. However, the limit $T_{n}^{d e g}=(1 / 3) T$ is not achieved for those ballistic lengths, since the samples are not sufficiently long. Note that $T_{n}$ is a measurable quantity, and the observation of $T_{n}$

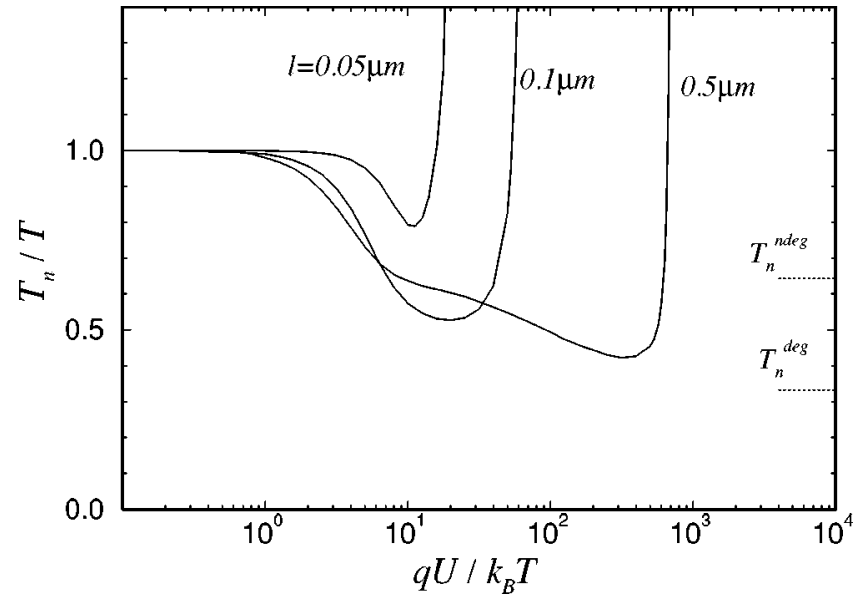

FIG. 6. Noise temperature $T_{n}$ vs bias $U$ for different ballistic lengths $l$. The asymptotic $(l \rightarrow \infty, U \rightarrow \infty)$ limits for nondegenerate and highly degenerate electrons are shown: $T_{n}^{n d e g}=3(1-\pi / 4) T$ and $T_{n}^{\text {deg }}=(1 / 3) T$, respectively.

$<T$ would indicate the significance of the Coulomb correlations effects which suppress the current noise. At $U \sim U_{c r}$, $T_{n}$ sharply increases due to the current saturation $(G=0)$, that may also be detected in the experiment.

The current-noise-suppression factors $\Gamma_{C}$ and $\Gamma_{F}$, and the total $\Gamma$ (their multiplication) correspondent to the noisepower curves of Fig. 4 are plotted in Fig. 7 as functions of bias. The behavior of the Fermi suppression factor $\Gamma_{F}$ is in a close agreement with the analytical formulas of Sec. VIII. It varies from 1 at low biases, then decreases attaining with a nice precision the asymptotic dependence $2 /\left(\alpha+3 \pi / \alpha^{2}\right)$ at high biases [Eq. (47)]. For all three cases, the same minimal value $2 / \xi \approx 0.2$ is reached at the highest biases, in agreement with the predictions. ${ }^{29}$ We have also checked that for the degenerate injection from both leads, which is well realized for the length $l=0.05 \mu \mathrm{m}$, the analytical formula [Eq. (51)] very nicely describes the numerical results in a wide bias range: from $U=0$ up to $U \sim 5 k_{B} T$ [Fig. 7(a)].

The relative significance of two mechanisms on the noise suppression can be understood by comparing the curves for three different ballistic lengths $l$. For short samples ( $l$ $=0.05 \mu \mathrm{m})$, the Fermi suppression dominates at low biases $V \lesssim 3$, where $\Gamma_{C} \approx 1$ and $\Gamma \approx \Gamma_{F}$ [see Fig. 7(a)]. At higher biases, $3 \leqq V \leqq 20$, both Coulomb and Fermi mechanisms contribute to the suppression. For $l=0.1 \mu \mathrm{m}$, the suppression factors $\Gamma_{F}$ and $\Gamma_{C}$ are comparable in all the bias range. Finally, for the longer sample, $l=0.5 \mu \mathrm{m}$, the Coulomb noise suppression completely dominates: $\Gamma_{C} \ll \Gamma_{F}$. This behavior can be explained by the fact that the Fermi shot-noisesuppression factor is limited below by the value $2 / \xi$, i.e., by the properties of the injecting contact independently of the ballistic length. ${ }^{29}$ In contrast, the Coulomb noise suppression may be enhanced arbitrarily strong by extending the length of the ballistic sample with a simultaneous increase of bias (provided the transport remains ballistic). Therefore, for any degree of electron degeneracy $\xi=\varepsilon_{F} / k_{B} T$, there exists the ballistic length starting from which the Coulomb interactions become to dominate in the noise suppression. 


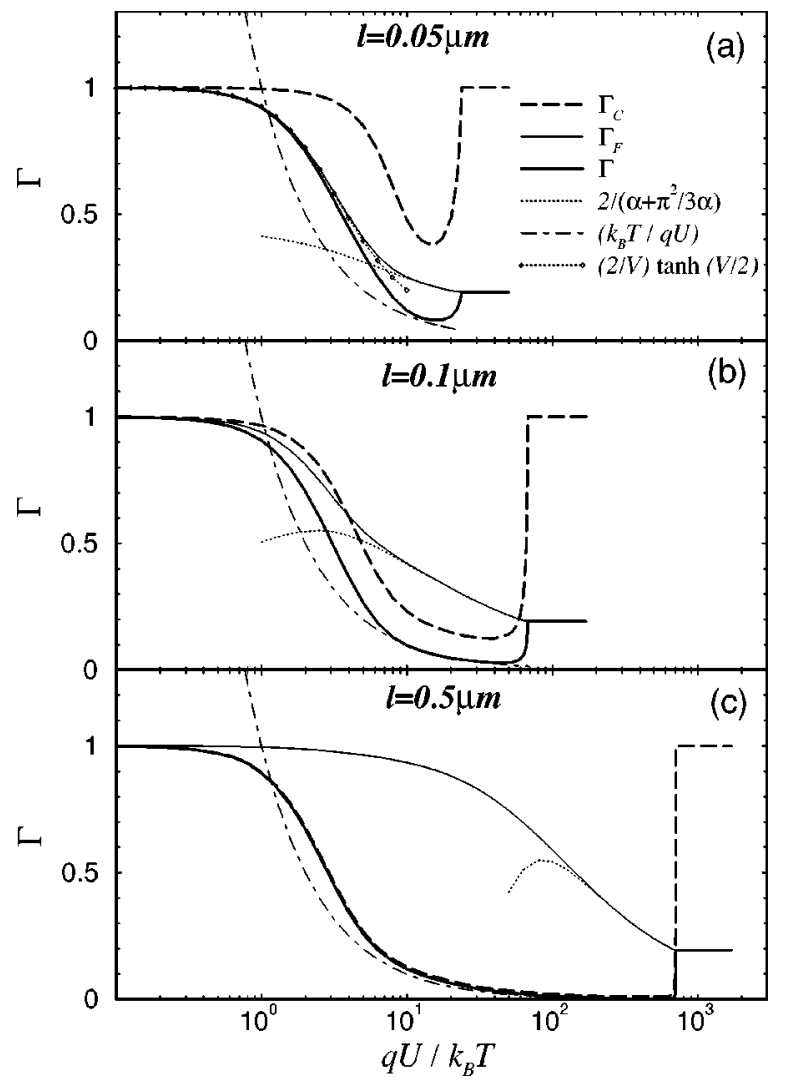

FIG. 7. Current-noise-suppression factors $\Gamma_{C}$ (Coulomb), $\Gamma_{F}$ (Fermi), and $\Gamma=\Gamma_{F} \Gamma_{C}$ (total) as functions of applied bias $U$ for different ballistic lengths $l$. The analytical approximations $2 /(\alpha$ $\left.+\pi^{2} / 3 \alpha\right)$ and $2 / V \tanh (V / 2)$ for $\Gamma_{F}$ and $k_{B} T / q U$ for $\Gamma$ are also shown.

Another important difference between the two suppression mechanisms is that the Fermi noise-suppression factor $\Gamma_{F}$ is a monotonically decreasing function of bias, while the Coulomb noise-suppression factor $\Gamma_{C}$ exhibits a minimum at a certain bias value, as seen in Fig. 7. After the minimum, the curve of $\Gamma_{C}$ increases to 1 due to the disappearance of the potential barrier at $U=U_{c r}$.

The total noise-suppression factor $\Gamma$ approaches at high biases the asymptotic curve $k_{B} T / q U$, once the injection, because of barrier lowering, becomes fully degenerate, in agreement with the prediction. ${ }^{29}$ The longer the sample, the wider is the bias range in which this asymptotic law is fulfilled independently of the material parameters (Fig. 7). It is also important that the suppression may be several orders of magnitude stronger than the shot-noise suppression due to elastic partitioning. ${ }^{3}$

It is instructive to plot the energy-resolved current-noise power $s(E)$ defined by $S_{I}=\int s(E) d E$. The derived formula [Eq. (36)] allows us to analyze these distributions for different lengths and biases. The results for $l=0.05$ and $0.5 \mu \mathrm{m}$ are shown in Figs. 8 and 9. At small biases $(V=0.1$ in the figures), the Coulomb interactions are ineffective, and the noise is approximately the sum of two equal contributions from the left and right leads. These contributions are the Fermi-Dirac profiles filled out above the barrier (the contributions of the reflected carriers with energies below the bar-

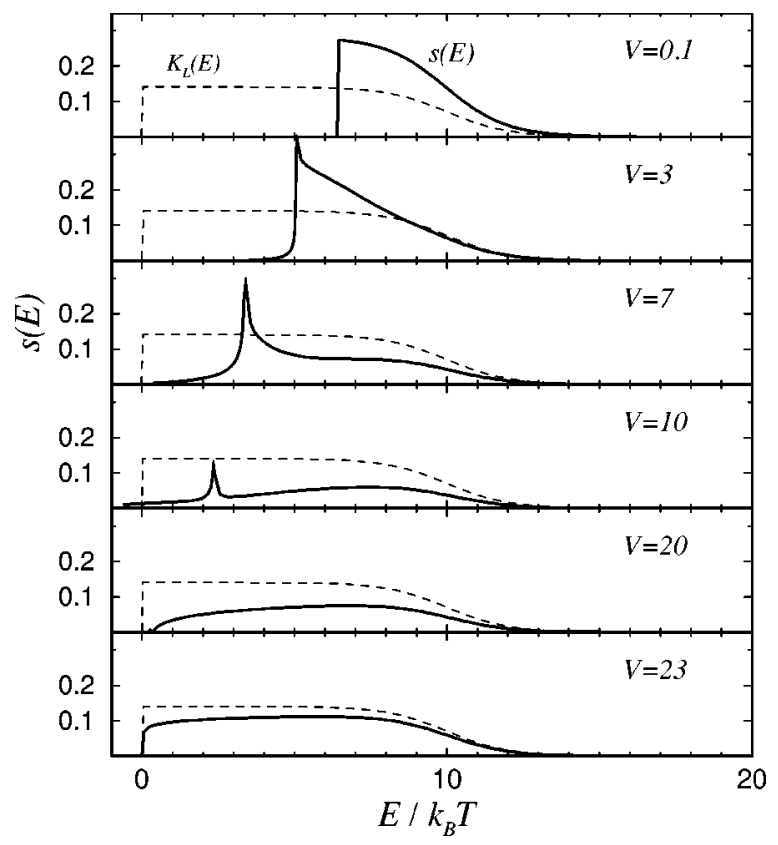

FIG. 8. Energy distributions of the current-noise power $s(E)$ under various biases $V$ for a ballistic conductor with $l=0.05 \mu \mathrm{m}$ (solid lines). The zero energy corresponds to the conduction-band edge at the left (unbiased) lead. The sharp peak at low energies corresponds to the position of the space-charge barrier. The dashed line shows the energy profile $K_{L}(E)$ for the injection noise at the left lead $\left(\varepsilon_{F}=10 k_{B} T\right)$. The profile for the right lead $K_{R}(E)$ is the same, but is shifted by $-V$ in energy. All the curves are normalized by $4 q I_{L}\left(\Phi_{L}^{0}\right)$ related to the noise level at $V=0$.

rier is negligible at these biases). With increasing the bias, several features appear: (i) the contribution from the right contact becomes smaller and smaller because of the shift in energy $-V$, (ii) the Coulomb interactions give rise to a sharp peak at the barrier energy with a noise suppression at the energies beyond the peak, and (iii) the carriers below the barrier give appreciable nonzero contribution to the noise. The peak appears due to the fact that electrons with the energy $E=\Phi_{L}$ virtually stop at the barrier top, producing an infinitely large perturbation of the current (this singularity is integrable, since it is of the logarithmic type ${ }^{18}$ ). Another interesting feature is the "noiseless" energy $E^{*}$ lying above the barrier, in which the noise exhibits a local minimum. It is better pronounced for a nondegenerate injection (see, for instance, Fig. 9) where one can observe the zero-noise point $s\left(E^{*}\right)=0$ for various biase). This point appears approximately at the condition: $\gamma_{L}\left(E^{*}\right)=0$. As long as the barrier vanishes at highest biases $U \rightarrow U_{c r}$, the Coulomb noise suppression disappears and the energy profile $s(E)$ recovers the FD shape.

\section{B. Fixed-current conditions: $\delta I=0$}

Thus far we have presented the results obtained under the assumption that the ballistic sample is connected to zeroimpedance external circuit. In this case the fluctuations of the applied voltage can be neglected. In experiments, it is the voltage fluctuations which are actually measured and which 


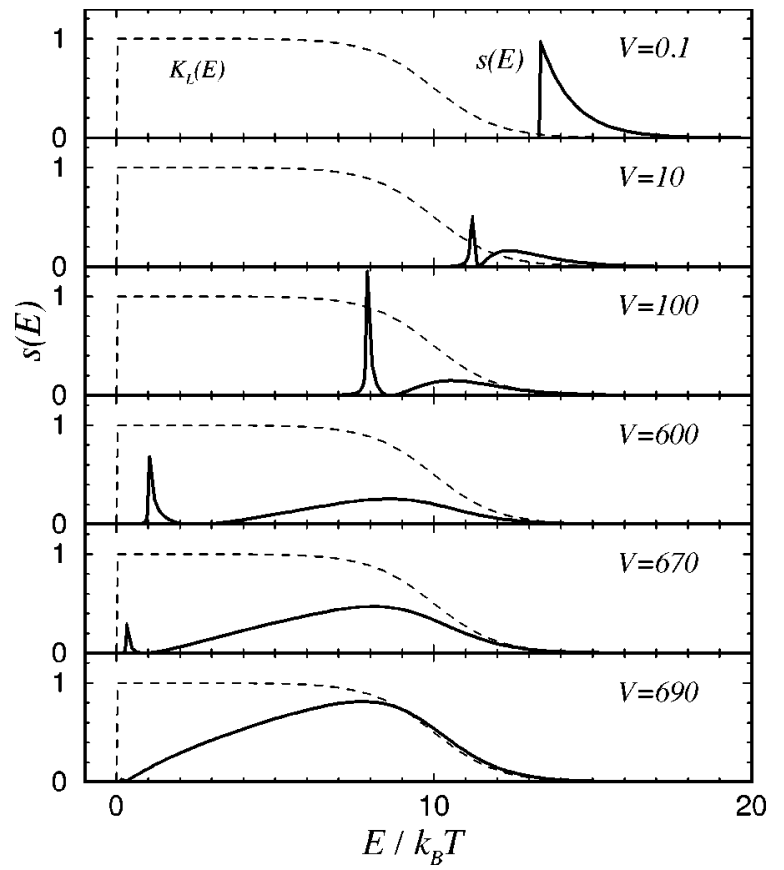

FIG. 9. Distributions similar to Fig. 8 for another ballistic length $l=0.5 \mu \mathrm{m}$. The notations are the same, except that $K_{L}(E)$ is not normalized.

eventually are converted to current fluctuations. By using an infinite-impedance circuit, the current fluctuations are forced to be zero, and one can analyze the voltage-noise power. Both cases are interrelated through Eq. (54). It is of interest however to see the results for the voltage-noise power-the quantity that can be measured directly.

Figure 10 shows the results of applying of Eq. (54) to our set of parameters. The behavior of $S_{U}$ calculated with and without Coulomb correlations is strikingly different. We remark the following features: (i) For the case when the interactions are included, the noise decreases with bias instead of increasing [the asymptotic behavior $S_{U} \sim 1 / \sqrt{U}$ at high biases (see Sec. X) is confirmed]. (ii) For longer samples, the range of the space-charge conduction is wider, and the suppression

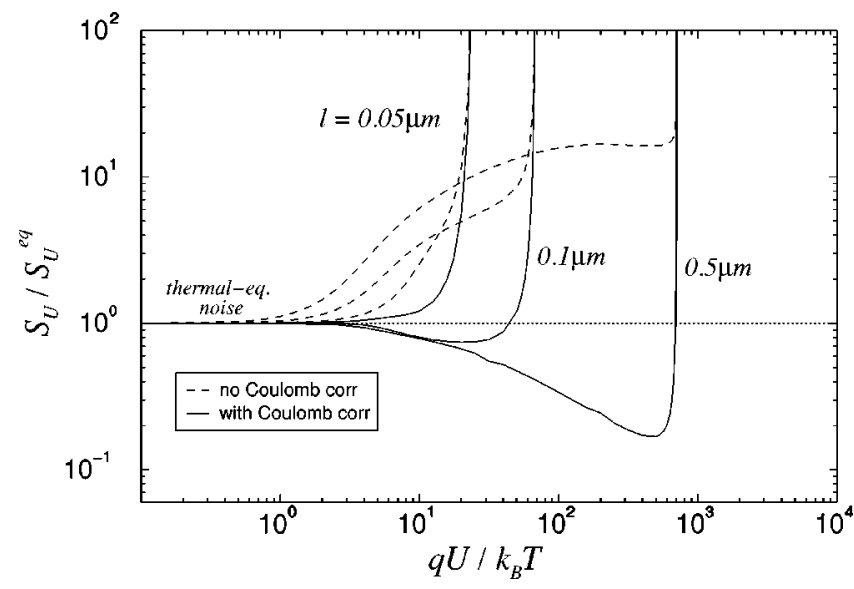

FIG. 10. Voltage-noise powers $S_{U}$ vs bias $U$ for different ballistic lengths $l$ are compared for two cases: with and without Coulomb correlations taken into account. of voltage fluctuations is much more pronounced. (iii) Comparing the asymptotic dependences $S_{U} \sim 1 / \sqrt{U}$ and $S_{I} \sim \sqrt{U}$, it is seen that the latter eventually exceeds the equilibrium Nyquist noise when the ballistic length is sufficiently long [see Fig. 4(c)]. In contrast, $S_{U}$ falls off the equilibrium value in a full range of SCL conduction. Note that the equilibrium fluctuations, described by the fluctuation-dissipation theorem, usually increase when an external electric field is applied. In our model, we have an interesting example when an interacting (via Coulomb forces) electron system is less noisy at far-from-equilibrium conditions than in equilibrium. For noninteracting electrons in quantum conductors, such examples have been provided by Lesovik and Loosen. ${ }^{21}$

We can also mention more familiar examples from semiconductor device literature. For instance, in Schottky-barrier diodes or $p$ - $n$ junctions, in the range of the exponential $I-V$ characteristics, the current-noise power is given by $S_{I}$ $=2 k_{B} T G$, which is a half of the thermal noise value given by the Nyquist relationship. ${ }^{34}$ However, this is not really a suppression effect, since the current-noise power $S_{I}$ never drops down the equilibrium Nyquist level $S_{I}^{e q}=4 k_{B} T G_{e q}$. In this case $G \gg G_{e q}$ and $S_{I}>S_{I}^{e q}$ for any bias ${ }^{35}$ (in our model $S_{I}<S_{I}^{e q}$ in a wide bias range). On the other hand, for these junctions under the fixed-current conditions, $S_{U}=2 k_{B} T G^{-1}$, that is again a half of the Nyquist relationship, but $S_{U}<S_{U}^{e q}$ may now occur. ${ }^{35}$ It should be emphasized, however, that the latter noise reduction appears for noninteracting carriers, and it is caused by the nonlinearity in the current-voltage characteristics which results in such a behavior that the conductance $G$ grows with bias as fast as the current-noise power $S_{I}$ (exponentially). As a result, $S_{U}=S_{I} / G^{2}$ is a decreasing function of bias. In our model, the noise suppression below the thermal equilibrium value (negative excess voltage noise) occurs due to Coulomb interactions among carriers. Without interactions, despite the nonlinear SCL regime, the noise grows above the Nyquist level, as was shown in Figs. 4 and 10.

\section{DISCUSSION}

In this paper, we have presented a theory of the electron transport and noise in a self-consistent potential along a ballistic two-terminal conductor. Since electrons are fermions and carry charge, they interact among themselves by both Fermi statistical correlations and long-range Coulomb correlations. The interplay of these two mechanisms determine the noise properties of a ballistic conductor-the subject we have addressed in the paper.

The long-range Coulomb correlations appear due to the self-consistent coupling between the electric potential and the occupation numbers. This coupling is essential to adequately describe the noise phenomena. To develop a better understanding of the Coulomb-correlation effect, we rewrite Eq. (1) — a standard equation for the mean current in a twoterminal conductor-in which we explicitly introduce the dependence of the transmission probabilities on the selfconsistent potential (in this case on the barrier height $\Phi_{b}$ ): 


$$
I=\int d \varepsilon\left[\mathcal{T}_{L}\left(\varepsilon, \Phi_{b}\right) I_{L}(\varepsilon)-\mathcal{T}_{R}\left(\varepsilon, \Phi_{b}\right) I_{R}(\varepsilon)\right] .
$$

Here, $I_{L, R}(\varepsilon)=(q A / 2 \pi \hbar) \int f_{L, R}\left(\varepsilon, \varepsilon_{\perp}\right) \nu_{\perp} d \varepsilon_{\perp}$ are the currents corresponding to the longitudinal energy $\varepsilon$. In the semiclassical limit, by neglecting the quantum-mechanical reflection of electrons with energies $\varepsilon>\Phi_{b}$ and tunneling through the barrier, the transmission probabilities are the Heaviside step functions: $\mathcal{T}_{L}\left(\varepsilon, \Phi_{b}\right)=\theta\left(\varepsilon-\Phi_{b}\right)$ and $\mathcal{T}_{R}\left(\varepsilon, \Phi_{b}\right)=\theta(\varepsilon$ $\left.-\Phi_{b}-q U\right)$.

The current fluctuation is found by perturbing Eq. (56); we obtain

$$
\begin{aligned}
\delta I= & \int\left[\mathcal{T}_{L} \delta I_{L}-\mathcal{T}_{R} \delta I_{R}\right] d \varepsilon \\
& +\int\left[\left(-\delta \Phi_{b}\right) \frac{\partial \mathcal{T}_{L}}{\partial \varepsilon} I_{L}+\left(-\delta \Phi_{b}-q \delta U\right) \frac{\partial \mathcal{T}_{R}}{\partial \varepsilon} I_{R}\right] d \varepsilon \\
\equiv & \delta I_{i n j}+\delta I_{\text {ind }},
\end{aligned}
$$

The first integral in Eq. (57) is the injected current fluctuation $\delta I_{i n j}$. Its origin is the thermal fluctuation of the occupation numbers in the leads ballistically injected into the conductor. In fact this term corresponds to Eq. (2) - the standard formula used to calculate the current noise in mesoscopic conductors. [Since for our case all $\mathcal{T}_{n}=0$ or 1 , the term $\propto \mathcal{T}_{n}\left(1-\mathcal{T}_{n}\right)$ is absent.] The second integral in Eq. (57) is the induced current fluctuation $\delta I_{\text {ind }}$ caused by the fluctuation of the potential. It is precisely the term appeared due to Coulomb correlations and ignored in Eq. (2). For our case, the derivatives are found as $\left(\partial \mathcal{T}_{L} / \partial \varepsilon\right)=\delta\left(\varepsilon-\Phi_{b}\right)$, $\left(\partial \mathcal{T}_{R} / \partial \varepsilon\right)=\delta\left(\varepsilon-\Phi_{b}-q U\right)$, leading to

$$
\delta I_{\text {ind }}=-\delta \Phi_{b} I_{L}\left(\Phi_{b}\right)+\left(\delta \Phi_{b}-q \delta U\right) I_{R}\left(\Phi_{b}+q U\right) .
$$

Thus Eqs. (57) and (58) lead to Eq. (27) for the current fluctuation derived more rigorously earlier from the transport equation.

We would like to highlight that the induced current fluctuations $\delta I_{\text {ind }}$ should appear not only in the case of completely open/closed channels $\left(\mathcal{T}_{n}=0 ; 1\right)$, but also under the conditions of the partitioning shot noise, for which there exist channels with $0<\mathcal{T}_{n}<1$. It is clear that $\delta I_{\text {ind }}$ should depend in general on the derivatives of the transmission probabilities $\left(\partial \mathcal{T}_{n} / \partial \varepsilon\right)$ and the fluctuations of the self-consistent potential $\delta \Phi_{x}$. The main problem is then to find the fluctuations $\delta \Phi_{x}$ through the noise sources. For the particular case of a multimode ballistic two-terminal conductor, we have found an exact analytical result for $\delta \Phi_{x}$. For the case of partitioning shot noise in which Eq. (2) holds, work is in progress.

The validity of our theory can be tested experimentally in currently accessible semiconductor structures. The required conditions are similar ${ }^{36}$ to those for the transport in vacuum tubes: (i) the ballistic electron transmission between the terminals, and (ii) the limitation of current by the space charge. The SCL transport regime, as applied for ballistic electrons in solids (mostly in $n^{+}-n-n^{+}$or $n-i-n$ semiconductor structures), was discussed a long time ago (see, e.g., theory ${ }^{37}$ and experiments ${ }^{38}$ ). Unfortunately, the data on noise measurements in these structures are scarce. ${ }^{39}$ Due to a great progress in noise measurements in quantum ballistic conductors during the last ten years ${ }^{7-10}$ (also see Ref. 3), we believe it would be now possible to measure the noise suppression effects in SCL ballistic conductors. Although the theoretical results presented in this paper are strictly valid for thick multichannel conductors (3D electron gas), the Coulomb suppression of noise should also be pronounced ${ }^{6}$ in conductors with a small number of channels (2D or 1D) in which electrons are more confined in space, for instance, in quantum wires under the high-bias nonlinear transport regime, ${ }^{40}$ or in carbon nanotubes under the SCL conduction. ${ }^{41}$

Additionally, we would like to emphasize the importance of the effect of Coulomb interactions. They not only lead to the noise reduction, but can also be used as a tool to probe the energy profile of the injected carriers and other electronic properties. $^{42}$

\section{ACKNOWLEDGMENTS}

This work was partially supported by the Ministerio de Ciencia y Tecnología of Spain through the "Ramón y Cajal" program.

\section{APPENDIX A: DERIVATION OF $d U_{b} / d U$}

Differentiating Eq. (13) gives

$$
\begin{aligned}
& \frac{d \Phi_{L}}{d U} \frac{1}{\sqrt{h_{-}\left(\Phi_{L}\right)}}-\frac{1}{2} \int_{0}^{\Phi_{L}} \frac{1}{h_{-}^{3 / 2}} \frac{d h_{-}}{d U} d \Phi \\
& \quad+\frac{d \Phi_{R}}{d U} \frac{1}{\sqrt{h_{+}\left(\Phi_{R}\right)}}-\frac{1}{2} \int_{0}^{\Phi_{R}} \frac{1}{h_{+}^{3 / 2}} \frac{d h_{+}}{d U} d \Phi=0 .
\end{aligned}
$$

By using $d \Phi_{R} / d U=q+d \Phi_{L} / d U$ and finding $d h / d U$ from Eq. (11), we obtain

$$
\frac{d h}{d U}=-\left(H_{L}+H_{R}\right) \frac{d \Phi_{L}}{d U}-q H_{R},
$$

where

$$
\begin{aligned}
& H_{L}(\Phi)= \frac{m}{2 \pi \hbar}\left\{\int_{0}^{\infty} \mathcal{D}_{L}[v(E+\Phi)-v(E)] d E\right. \\
&\left.+2 \theta(-\chi) \int_{-\Phi}^{0} \mathcal{D}_{L} v(E+\Phi) d E\right\}, \\
& H_{R}(\Phi)= \frac{m}{2 \pi \hbar}\left\{\int_{0}^{\infty} \mathcal{D}_{R}[v(E+\Phi)-v(E)] d E\right. \\
&\left.+2 \theta(\chi) \int_{-\Phi}^{0} \mathcal{D}_{R} v(E+\Phi) d E\right\}, \\
& \mathcal{D}_{k}(E)=-\left.\frac{\partial n_{k}}{\partial E}\right|_{E+\Phi_{k}}, \quad k=L, R .
\end{aligned}
$$

Substituting Eqs. (A2)-(A4) into Eq. (A1), we finally obtain 


$$
\frac{d U_{b}}{d U}=\frac{1}{q} \frac{d \Phi_{L}}{d U}=-\frac{\Delta_{R}}{\Delta_{L}+\Delta_{R}} .
$$

Here we have defined

$$
\begin{aligned}
& \Delta_{L}=\frac{2}{\sqrt{h_{-}\left(\Phi_{L}\right)}}+\int_{0}^{\Phi_{L}} \frac{H_{L}^{-}}{h_{-}^{3 / 2}} d \Phi+\int_{0}^{\Phi_{R}} \frac{H_{L}^{+}}{h_{+}^{3 / 2}} d \Phi, \\
& \Delta_{R}=\frac{2}{\sqrt{h_{+}\left(\Phi_{R}\right)}}+\int_{0}^{\Phi_{L}} \frac{H_{R}^{-}}{h_{-}^{3 / 2}} d \Phi+\int_{0}^{\Phi_{R}} \frac{H_{R}^{+}}{h_{+}^{3 / 2}} d \Phi,
\end{aligned}
$$

where we have denoted $H_{k}^{-} \equiv H_{k}(\chi<0)$ and $H_{k}^{+} \equiv H_{k}(\chi$ $>0), k=L, R$. The quantities $\Delta_{L}$ and $\Delta_{R}$, as well as their sum

$$
\Delta=\Delta_{L}+\Delta_{R},
$$

are used in this paper to calculate the differential conductance [Eq. (21)] and the noise suppression [Eq. (30)]. The physical meaning of $\Delta$ becomes clear from the relation

$$
\Delta=2 q \sqrt{\frac{2}{\kappa}}\left(\frac{\partial l}{\partial \Phi_{L}}\right)_{U=\text { const }},
$$

i.e., it relates the increment of the barrier height with the increase of the length of the sample under fixed bias. $\Delta_{L, R}$ are the corresponding contributions to that increment from the left-lead and right-lead electrons.

\section{APPENDIX B: DERIVATION OF THE SELF-CONSISTENT POTENTIAL FLUCTUATIONS}

Integrating the fluctuation of the occupation factor $\delta n(E)$ over the longitudinal states, one obtains the electron-density fluctuation as a sum of two contributions, $\delta N=\delta N^{i n j}$ $+\delta N^{\text {ind }}$, where the injected part is

$$
\begin{aligned}
\delta N^{i n j}(\Phi)= & \int_{0}^{\infty}\left[\delta n_{L}\left(E+\Phi_{L}\right)+\delta n_{R}\left(E+\Phi_{R}\right)\right] \nu(E+\Phi) d E \\
& +2 \int_{-\Phi}^{0}\left[\theta(-\chi) \delta n_{L}\left(E+\Phi_{L}\right)\right. \\
& \left.+\theta(\chi) \delta n_{R}\left(E+\Phi_{R}\right)\right] \nu(E+\Phi) d E
\end{aligned}
$$

and the induced part is

$$
\delta N^{i n d}(\Phi)=\frac{d N}{d \Phi} \delta \Phi_{x}-\frac{d H_{L}}{d \Phi} \delta \Phi_{L}-\frac{d H_{R}}{d \Phi} \delta \Phi_{R} .
$$

Equations (B1) and (B2) should now be substituted into the Poisson equation for $\delta \Phi_{x}$ to find the self-consistent fluctuation of the potential profile:

$$
\frac{d^{2}}{d x^{2}} \delta \Phi_{x}=\frac{q^{2}}{\kappa}\left(\delta N^{i n j}+\delta N^{i n d}\right) .
$$

We obtain

$$
\hat{L} \delta \Phi_{x} \equiv\left[\frac{d^{2}}{d x^{2}}-\frac{q^{2}}{\kappa} \frac{d N}{d \Phi}\right] \delta \Phi_{x}=\delta s_{x}
$$

where $\quad \delta s_{x}=\left(q^{2} / \kappa\right)\left[\delta N^{i n j}-\left(d H_{L} / d \Phi\right) \delta \Phi_{L}-\left(d H_{R} /\right.\right.$ $\left.d \Phi) \delta \Phi_{R}\right]$. The boundary conditions for this equation $\delta \Phi_{x}(0)=\delta \Phi_{L}, \delta \Phi_{x}(l)=\delta \Phi_{R}, \delta \Phi_{x}\left(x_{b}\right)=0$.

The second-order differential equation (B4) with spatially dependent coefficients can be solved explicitly for $\delta \Phi_{x} .{ }^{18}$ Here we need just the boundary values $\delta \Phi_{L}$ and $\delta \Phi_{R}$ (the relation between them), which has entered explicitly into the nonhomogeneous part and can be obtained by applying the Green's identity for the operator $\hat{L}$,

$$
\int_{a}^{b}\left[u(x) \hat{L} \delta \Phi_{x}-\delta \Phi_{x} \hat{L} u(x)\right] d x=\left.\left(u(x) \frac{d \delta \Phi}{d x}-\delta \Phi_{x} \frac{d u}{d x}\right)\right|_{a} ^{b},
$$

where $[a ; b]=\left[0 ; x_{b}\right]$ for $\chi<0$ and $[a ; b]=\left[x_{b} ; l\right]$ for $\chi$ $>0$. It is convenient to chose the function $u(x)$ as a solution of the homogeneous equation $\hat{L} u(x)=0$ satisfying the boundary conditions $u(0)=0$ and $u(l)=0$. This gives

$$
\int_{0}^{x_{b}} u \delta s_{x} d x+\int_{x_{b}}^{l} u \delta s_{x} d x=\frac{\delta \Phi_{L}}{\mathcal{E}_{L}}-\frac{\delta \Phi_{R}}{\mathcal{E}_{R}},
$$

where $\mathcal{E}_{L}$ and $\mathcal{E}_{R}$ are the electric fields at $x=0$ and $x=l$, respectively. Changing the variable of integration $d x$ $=-d \Phi /(q \mathcal{E})$, one obtains

$$
\int_{0}^{\Phi_{L}} \frac{u}{\mathcal{E}} \delta s_{x} d \Phi-\int_{0}^{\Phi_{R}} \frac{u}{\mathcal{E}} \delta s_{x} d \Phi=\frac{\delta \Phi_{L}}{\mathcal{E}_{L}}-\frac{\delta \Phi_{R}}{\mathcal{E}_{R}}
$$

It is convenient to represent the fluctuation $\delta s_{x}$ as a derivative $\delta s_{x}=\left(\kappa / q^{2}\right)(d \delta h / d \Phi)$. By using this notation, the integrals in Eq. (B6) can be reduced to ${ }^{27}$

$$
\int_{0}^{\Phi_{k}} \frac{u}{\mathcal{E}} \frac{d \delta h}{d \Phi} d \Phi=\frac{1}{q} \int_{0}^{\Phi_{k}} \frac{\delta h}{\mathcal{E}^{3}} d \Phi, \quad k=L, R
$$

whereas $\delta h$ is obtained by integration of $\delta s_{x}$ :

$$
\delta h=\delta h^{i n j}-H_{L} \delta \Phi_{L}-H_{R} \delta \Phi_{R},
$$

$$
\delta h^{i n j}(\Phi)=\int_{0}^{\Phi} \delta N^{i n j} d \Phi
$$

Now substituting Eqs. (B7) and (B8) into Eq. (B6), and by using Eq. (10), we obtain

$$
\Delta \delta \Phi_{L}+\Delta_{R} q \delta U=\int_{0}^{\Phi_{L}} \frac{\delta h_{-}^{i n j}}{h_{-}^{3 / 2}} d \Phi+\int_{0}^{\Phi_{R}} \frac{\delta h_{+}^{i n j}}{h_{+}^{3 / 2}} d \Phi,
$$

where $\Delta$ and $\Delta_{R}$ were denoted in Appendix A. Combining Eqs. (27) and (B10) and excluding $\delta \Phi_{L}$, we obtain 


$$
\begin{aligned}
\delta I- & G \delta \\
= & \int_{0}^{\infty}\left[\delta I_{L}\left(E+\Phi_{L}\right)-\delta I_{R}\left(E+\Phi_{R}\right)\right] d E \\
& -\frac{I_{L}\left(\Phi_{L}\right)-I_{R}\left(\Phi_{R}\right)}{\Delta}\left(\int_{0}^{\Phi_{L}} \frac{\delta h_{-}^{i n j}}{h_{-}^{3 / 2}} d \Phi+\int_{0}^{\Phi_{R}} \frac{\delta h_{+}^{i n j}}{h_{+}^{3 / 2}} d \Phi\right),
\end{aligned}
$$

that leads to Eq. (28).

\section{APPENDIX C: NYQUIST THEOREM AND THE BOUNDARY CONDITIONS FOR FLUCTUATIONS}

Consider the situation when the potentials at the leads are held equal $(U=0, \delta U=0)$ by means of a zero-impedance external circuit. (A similar consideration can be carried out for the infinite-impedance circuit.) Additionally we assume that the contacts are identical: $I_{L}(E)=I_{R}(E)$, and $K_{L}(E)$ $=K_{R}(E), \forall E$. Thus from Eq. (30) we have $C_{\Delta}=0, \gamma_{L}(E)$ $=\theta(E)$, and $\gamma_{R}(E)=-\theta(E)$, which means that Coulomb correlations do not affect noise at zero bias. Therefore, from Eqs. (28) and (33) one obtains the current-noise power

$$
S_{I}^{e q}=2 \int_{0}^{\infty} K_{L}\left(E+\Phi_{L}^{0}\right) d E
$$

where $\Phi_{L}^{0}$ is the equilibrium barrier height (the noise depends on the steady-state self-consistent field). For the equilibrium conductance we find

$$
G_{e q}=\left.\frac{d I}{d U}\right|_{U=0}=-\left.q \int_{0}^{\infty} \frac{\partial I_{L}}{\partial E}\right|_{E+\Phi_{L}^{0}} d E .
$$

By using the Nyquist theorem $S_{I}^{e q}=4 k_{B} T G_{e q}$, we obtain

$$
\int_{0}^{\infty} K_{L}\left(E+\Phi_{L}^{0}\right) d E=\left.2 q k_{B} T \int_{0}^{\infty}\left(-\frac{\partial I_{L}}{\partial E}\right)\right|_{E+\Phi_{L}^{0}} d E .
$$

Since this integral relation should be valid for different lengths $l$ of the ballistic conductor (different $\Phi_{L}^{0}$ ), it should also be valid for the integrands,

$$
K_{L}(E)=2 q k_{B} T\left(-\frac{\partial I_{L}}{\partial E}\right),
$$

that leads to Eq. (34). Thus, just from the Nyquist theorem we have a useful relation for the energy-resolved currents (occupation factors) at the leads. It relates the energy profiles of the fluctuations and the mean values. In the simplest case of the Poissonian injection, for instance, the correlation function is proportional to the mean ${ }^{11}$ :

$$
K_{L}^{\text {Pois }}(E)=2 q I_{L}(E) .
$$

From this result it follows that $I_{L}(E)=-k_{B} T\left(\partial I_{L} / \partial E\right)$, and one obtains the Boltzmann distribution

$$
I_{L}^{\mathrm{Pois}}(E)=C \exp \left(-E / k_{B} T\right),
$$

where the integration constant $C$ is determined by the normalization condition.
*Electronic address: oleg@ffn.ub.es

${ }^{1}$ M.J.M. de Jong and C.W.J. Beenakker, in Mesoscopic Electron Transport, edited by L.P. Kowenhoven, G. Schön, and L.L. Sohn (Kluwer, Dordrecht, 1997), p. 225.

${ }^{2}$ R. Landauer, Nature (London) 392, 658 (1998).

${ }^{3}$ Ya.M. Blanter and M. Büttiker, Phys. Rep. 336, 1 (2000).

${ }^{4}$ G.B. Lesovik, Pis'ma Zh. Éksp. Teor. Fiz. 49, 513 (1989) [JETP Lett. 49, 592 (1989)].

${ }^{5}$ M. Büttiker, Phys. Rev. Lett. 65, 2901 (1990); Phys. Rev. B 46, 12485 (1992).

${ }^{6}$ R. Landauer and Th. Martin, Physica B 175, 167 (1991); Th. Martin and R. Landauer, Phys. Rev. B 45, 1742 (1992).

${ }^{7}$ Y.P. Li, D.C. Tsui, J.J. Heremans, J.A. Simmons, and G.W. Weimann, Appl. Phys. Lett. 57, 774 (1990).

${ }^{8}$ M. Reznikov, M. Heiblum, H. Shtrikman, and D. Mahalu, Phys. Rev. Lett. 75, 3340 (1995).

${ }^{9}$ A. Kumar, L. Saminadayar, D.C. Glattli, Y. Jin, and B. Etienne, Phys. Rev. Lett. 76, 2778 (1996).

${ }^{10}$ H.E. van den Brom and J.M. van Ruitenbeek, Phys. Rev. Lett. 82, 1526 (1999).

${ }^{11}$ Sh. Kogan, Electronic Noise and Fluctuations in Solids (Cambridge University Press, Cambridge, 1996), Chap. 5.

${ }^{12}$ In Eq. (3), $S_{I}$ depends on the bias through the parameter $n^{*}$, i.e., the number of open channels which may vary with bias. The same is true for the current. In the absence of partitioning, the current varies with bias due to the space-charge impediment.

${ }^{13}$ R. Landauer, Physica D 38, 226 (1989).
${ }^{14}$ See, for instance, the recent study by V.A. Sablikov and B.S. Shchamkhalova, Phys. Rev. B 58, 13847 (1998).

${ }^{15}$ V.A. Sablikov, S.V. Polyakov, and M. Büttiker, Phys. Rev. B 61, 13763 (2000)

${ }^{16}$ M. Büttiker, Pramana J. Phys. 58, 241 (2002).

${ }^{17}$ Y. Wei, B. Wang, J. Wang, and H. Guo, Phys. Rev. B 60, 16900 (1999).

${ }^{18}$ O.M. Bulashenko, J.M. Rubí, and V.A. Kochelap, Phys. Rev. B 61, 5511 (2000).

${ }^{19}$ R. Landauer, Phys. Rev. B 47, 16427 (1993); Physica B 227, 156 (1996).

${ }^{20}$ M. Büttiker, J. Math. Phys. 37, 4793 (1996).

${ }^{21}$ G.B. Lesovik and R. Loosen, Z. Phys. B: Condens. Matter 91, 531 (1993)

${ }^{22}$ A.G. Scherbakov, E.N. Bogachek, and U. Landman, Phys. Rev. B 57, 6654 (1998).

${ }^{23}$ A.F.J. Levi, J.R. Hayes, P.M. Platzman, and W. Wiegmann, Phys. Rev. Lett. 55, 2071 (1985).

${ }^{24}$ M. Heiblum, M.I. Nathan, D.C. Thomas, and C.M. Knoedler, Phys. Rev. Lett. 55, 2200 (1985).

${ }^{25}$ M. Kast, C. Pacher, G. Strasser, and E. Gornik, Appl. Phys. Lett. 78, 3639 (2001).

${ }^{26}$ C. Strahberger, J. Smoliner, R. Heer, and G. Strasser, Phys. Rev. B 63, 205306 (2001).

${ }^{27}$ O.M. Bulashenko, J.M. Rubí, and V.A. Kochelap, Phys. Rev. B 62, 8184 (2000).

${ }^{28}$ Equation (13) is valid for the biases $U<U_{c r}$ when the barrier 
exists, where $U_{c r}$ is the bias for which $d \Phi / d x=0$ at $x=0$. The case $U>U_{c r}$ is not interesting, since the transport is no longer space-charge limited, and the noise is no longer suppressed by Coulomb correlations.

${ }^{29}$ O.M. Bulashenko and J.M. Rubí, Phys. Rev. B 64, 045307 (2001).

${ }^{30}$ A. van der Ziel, Noise in Solid State Devices and Circuits (Wiley, New York, 1986), Chap. 3.1.

${ }^{31}$ P. Shiktorov, V. Gruzinskis, E. Starikov, L. Reggiani, and L. Varani, Phys. Rev. B 54, 8821 (1996).

${ }^{32}$ O.M. Bulashenko, J.M. Rubí, and V.A. Kochelap, Appl. Phys. Lett. 75, 2614 (1999).

${ }^{33}$ In $n-i-n$ structures, the current is mostly carried by electrons injected from the contacts ( $n$ regions). The contribution of intrinsic carriers of the sample ( $i$ region) is negligible. Similar calculations can be carried out for $n^{+}-n-n^{+}, n^{+}-p-n^{+}$diodes, heterodiodes (Ref. 32) and others.

${ }^{34}$ A. van der Ziel, Noise in Solid State Devices and Circuits (Ref. 30), Chap. 6.1.

${ }^{35}$ In Schottky-barrier diodes and $p$ - $n$ junctions, the mean current is a sum of two opposite current flows crossing the barrier: $I=I_{L}$ $-I_{R}$, with $I_{L}=I_{0} \exp \left(q U / k_{B} T\right)$ and $I_{R}=I_{0}$ (see Ref. 34). Hence the conductance is $G=q\left(I+I_{0}\right) / k_{B} T$, assuming $I_{0}$ is a slow function of $U$. Neglecting correlations among carriers, the current noise power is a sum of two Poissonian noise terms: $S_{I}$ $=2 q\left(I_{L}+I_{R}\right)=2 q\left(I+2 I_{0}\right)=2 k_{B} T G\left[\left(I+2 I_{0}\right) /\left(I+I_{0}\right)\right]$. The nonequilibrium noise at high currents $I \gg I_{0}$ is $S_{I}=2 k_{B} T G$, which is a half of the Nyquist thermal noise of a resistor having the identical (nonequilibrium) conductance $G$. However, by re- writing $S_{I}=S_{I}^{e q}\left[1+I /\left(2 I_{0}\right)\right]$, it is easy to see that $S_{I}>S_{I}^{e q}$ for any $I$. On the other hand, the voltage-noise power $S_{U}=S_{I} / G^{2}$ $=S_{U}^{e q}\left[\left(1+I / 2 I_{0}\right) /\left(1+I / I_{0}\right)^{2}\right]$ is lower than the equilibrium $\mathrm{Ny}$ quist value $S_{U}^{e q}$, since it decreases with current as $S_{U}$ $\approx S_{U}^{e q}\left(I_{0} / 2 I\right)$.

${ }^{36}$ The distinction between the electron transport in ballistic solidstate and vacuum diodes was drawn in Refs. 18 and 32.

${ }^{37}$ M.S. Shur and L.F. Eastman, IEEE Trans. Electron Devices 26, 1677 (1979); A.J. Holden and B.T. Debney, Electron. Lett. 18, 558 (1982); H.U. Baranger and J.W. Wilkins, Phys. Rev. B 30, 7349 (1984).

${ }^{38}$ L.F. Eastman, R. Stall, D. Woodard, N. Dandekar, C.E.C. Wood, M.S. Shur, and K. Board, Electron. Lett. 16, 524 (1980); M.A. Hollis, L.F. Eastman, and C.E.C. Wood, ibid. 18, 524 (1982); more recently, E. Hernández, Cryst. Res. Technol. 33, 285 (1998).

${ }^{39}$ R.R. Schmidt, G. Bosman, C.M. Van Vliet, L.F. Eastman, and M. Hollis, Solid-State Electron. 26, 437 (1983).

${ }^{40}$ K.S. Novoselov, Yu.V. Dubrovskii, V.A. Sablikov, D.Yu. Ivanov, E.E. Vdovin, Yu.N. Khanin, V.A. Tulin, D. Esteve, and S. Beaumont, Europhys. Lett. 52, 660 (2000).

${ }^{41}$ Z.K. Tang, H.D. Sun, J. Wang, J. Chen, and G. Li, Appl. Phys. Lett. 73, 2287 (1998); Ph. Avouris, R. Martel, H. Ikeda, M. Hersam, H.R. Shea, and A. Rochefort, in Science and Application of Nanotubes, edited by D. Tománek and R. J. Enbody (Kluwer, New York, 2000), p. 223.

${ }^{42}$ O.M. Bulashenko and J.M. Rubí, Physica E (Amsterdam) 12, 857 (2002). 